\title{
AT 2016dah and AT 2017fyp: the first classical novae discovered within a tidal stream
}

\author{
M. J. Darnley ${ }^{\oplus},{ }^{\star}$ A. M. Newsam, ${ }^{1 \star}$ K. Chinetti, ${ }^{1,2}$ I. D. W. Hawkins, ${ }^{1}$ \\ A. L. Jannetta, ${ }^{1,3}$ M. M. Kasliwal ${ }^{\oplus},{ }^{2}$ J. C. M $^{\mathrm{c}}$ Garry, ${ }^{1,4}$ M. M. Shara, ${ }^{5}$ M. Sitaram ${ }^{1,6}$ \\ and S. C. Williams ${ }^{\oplus 7,8,9}$
}

${ }^{1}$ Astrophysics Research Institute, Liverpool John Moores University, IC2 Liverpool Science Park, Liverpool L3 5RF, UK

${ }^{2}$ Division of Physics, Mathematics and Astronomy, California Institute of Technology, Pasadena, CA 91125, USA

${ }^{3}$ INTO Newcastle University, The INTO Building, Newcastle University, Newcastle upon Tyne NE1 7RU, UK

${ }^{4}$ Centre for Astrophysics Research, University of Hertfordshire, College Lane, Hatfield AL10 9AB, UK

${ }^{5}$ Department of Astrophysics, American Museum of Natural History, 79th Street and Central Park West, New York, NY 10024, USA

${ }^{6}$ Department of Astronomy, University of Maryland, College Park, MD 20742-2421, USA

${ }^{7}$ Finnish Centre for Astronomy with ESO (FINCA), University of Turku, Quantum, Vesilinnantie 5, 20014 Turku, Finland

${ }^{8}$ Department of Physics and Astronomy, University of Turku, 20014 Turku, Finland

${ }^{9}$ Physics Department, Lancaster University, Lancaster LA1 4YB, UK

Accepted 2020 April 20. Received 2020 April 20; in original form 2020 March 10

\begin{abstract}
AT 2016dah and AT 2017fyp are fairly typical Andromeda galaxy (M31) classical novae. AT 2016dah is an almost text book example of a 'very fast' declining, yet uncommon, Fe II 'b' (broad-lined) nova, discovered during the rise to peak optical luminosity, and decaying with a smooth broken power-law light curve. AT 2017fyp is classed as a 'fast' nova, unusually for M 31, its early decline spectrum simultaneously shows properties of both $\mathrm{Fe}$ II and $\mathrm{He} / \mathrm{N}$ spectral types - a 'hybrid'. Similarly, the light curve of AT 2017fyp has a broken power-law decline but exhibits an extended flat-topped maximum. Both novae were followed in the UV and X-ray by the Neil Gehrels Swift Observatory, but no X-ray source was detected for either nova. The pair were followed photometrically and spectroscopically into their nebular phases. The progenitor systems were not visible in archival optical data, implying that the mass donors are main-sequence stars. What makes AT 2016dah and AT 2017fyp particularly interesting is their position with respect to M31. The pair are close on the sky but are located far from the centre of M 31, lying almost along the semiminor axis of their host. Radial velocity measurements and simulations of the M 31 nova population leads to the conclusion that both novae are members of the Andromeda Giant Stellar Stream (GSS). We find the probability of at least two M 31 novae appearing coincident with the GSS by chance is $\sim 1$ per cent. Therefore, we claim that these novae arose from the GSS progenitor, not M 31 - the first confirmed novae discovered in a tidal steam.
\end{abstract}

Key words: stars: individual: (AT 2016dah, AT 2017fyp)-novae, cataclysmic variables galaxies: haloes - galaxies: individual: M31 - ultraviolet: stars.

\section{INTRODUCTION}

Almost half a century ago, Toomre \& Toomre (1972) published a classic, monumental paper that forever changed how astronomers think about the formation and evolution of galaxies. Their simpletitled paper 'Galactic Bridges and Tails' demonstrated that previously unexplained, luminous connections between galaxies and

^E-mail: M.J.Darnley@1jmu.ac.uk (MDJ); A.Newsam@1jmu.ac.uk (ANM) long streamers emanating from those galaxies are tidal in origin. Their tour de force fig. 23 shows a model of NGC 4038 and NGC 4039, also known as 'The Antennae', which mimics those galaxies' tidal tails with remarkable fidelity. The tails stretch far beyond the confines of each of the galaxies; the stars in them will never return to the galaxies in which they were born.

Rather than being arcane, evanescent features of galaxies sweeping by or through each other, tails and bridges highlight the changes in masses, sizes, morphologies, and star-forming histo- 
ries of galaxies that shape the appearances of the galaxies we observe today. During close galaxy-galaxy encounters, a fraction of the stars in each galaxy acquire sufficient kinetic energy to permanently escape into intergalactic space, thereby becoming 'escaped' or hostless stars. Others travel to further than a few virial radii for longer than a few Gyr, but still remain energetically bound to their parent galaxy, becoming 'wandering' stars (Teyssier, Johnston \& Shara 2009). The detection of these hostless and of wandering stars, and determination of their numbers and spatial distributions, is an important constraint on tidal stripping efficiency. Miller (1983) and Dressler (1984) stressed the importance of obtaining reliable measurements of the intracluster light as a direct indicator of the tidal damage suffered by galaxies.

Clusters of galaxies are targets amenable to searches for such intracluster stars. Hostless, or intracluster, planetary nebulae $(\mathrm{PNe})$ are particularly attractive for intracluster star searches, and they have been detected, via their strong [O III] emission, in multiple clusters, including Fornax (Theuns \& Warren 1997), Virgo (Feldmeier, Ciardullo \& Jacoby 1998; Longobardi et al. 2013), and Coma (Gerhard et al. 2005). Hostless type Ia supernovae have been employed as probes to indirectly measure the fraction of intracluster light (see for e.g. Gal-Yam et al. 2003; McGee \& Balogh 2010; Sand et al. 2011; Graham et al. 2015).

Classical novae (CNe; see for e.g. Bode \& Evans 2008; Woudt \& Ribeiro 2014, for recent compilations of reviews) are up to $100 \times$ more luminous than $\mathrm{PNe}$, and so sample a volume $1000 \times$ larger. $\mathrm{CNe}$ call attention to themselves both by their 10 20 mag amplitude eruptions and their strong and persisting $\mathrm{H} \alpha$ emission lines (see, for e.g. Ciardullo et al. 1987). Intracluster CNe have been detected in the Fornax galaxy cluster (Neill, Shara \& Oegerle 2005).

The spatial distribution of those Fornax intracluster novae is consistent with $\sim 28 \pm 13$ per cent of the total light in the cluster being in the intracluster light (Neill et al. 2005). Similar fractions are evident from the Fornax PNe, while the deepest recent searches in Virgo yield an estimate of 7-15 per cent for the fraction of intracluster light in that cluster (Mihos et al. 2017). This demonstrates that intracluster stars are a significant fraction of the stars in clusters, and of course their fraction will grow monotonically in the Gyr to come.

Our knowledge of hostless and wandering stars outside clusters is sparse. That they exist in the environs of the Milky Way and in the Local Group is evident from distant M-giant surveys (Bochanski et al. 2014), the existence of the RR Lyrae and M-giant tracers in the Sagittarius Stream (Ibata et al. 2001b) and Magellanic Stream (D'Onghia \& Fox 2016), and the stellar streams of the Andromeda Galaxy (M31), particularly its Giant Stellar Stream (hereafter GSS; Ibata et al. 2001a). The size of the Local Group intracluster population is almost certainly smaller than that in rich clusters of galaxies, but there is currently no quantitative estimate of that size.

The ease with which erupting $\mathrm{CNe}$ can be detected with 1- and 2-m telescopes, which are now routinely carrying out automated wide-field CCD transient surveys, means that they are prime candidates for mapping hostless and wandering stars out to at least 3-5 Mpc (Shara 2006) - the M 81 galaxy group and beyond. The neighbourhood of M31, which is being heavily targeted by several transient surveys, is a particularly useful target because the transient population in that galaxy, particularly its nova content (see Darnley \& Henze 2019; Shafter 2019, for recent reviews), is extremely well-studied.

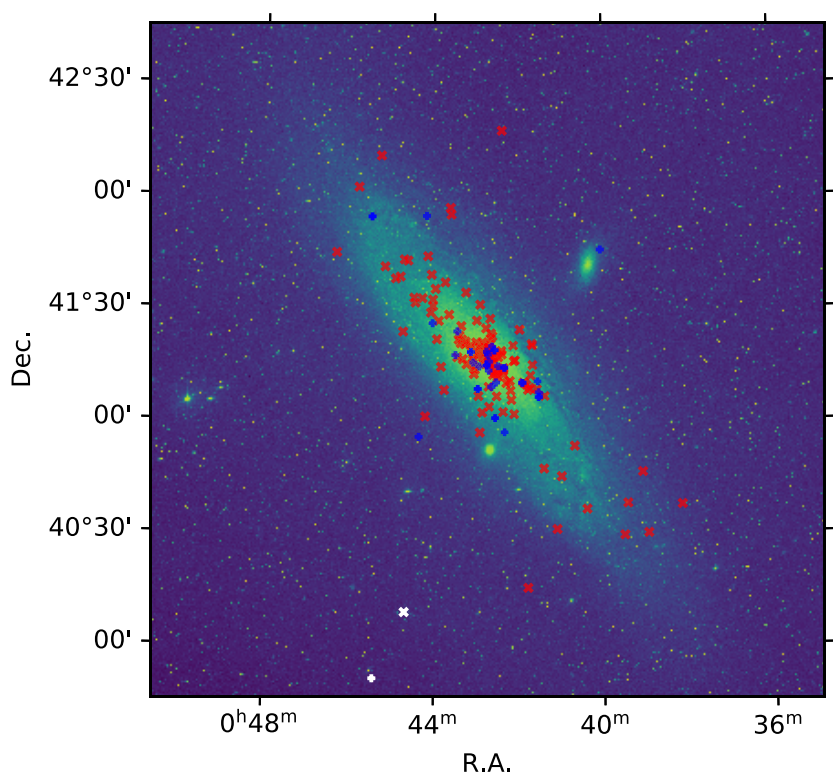

Figure 1. False-colour Digitized Sky Survey (DSS; Lasker et al. 1990) red mosaic of M 31 overplotted with the positions of 135 spectroscopically confirmed M 31 Fe II novae ( $\mathrm{red} \times$ ) and $38 \mathrm{He} / \mathrm{N}$ (including hybrids) novae (blue +); spectroscopic data from Shafter et al. (2011, and Ransome et al in preparation). The white data points show the location of AT 2016dah (northern most; $\times$ ) and AT 2017fyp (southern most; +).

Here, we report on a pair of $\mathrm{CNe}$ discovered 1.2 and 1.5 from the centre of M31, close to the minor axis of their highly inclined host, and far beyond its visible optical disc. Both appear to be strongly associated with the M31 GSS (see Ibata et al. 2001a, and Section 4). Dozens more such detections will be needed to fully map out the hostless and wandering $\mathrm{CNe}$ associated with $\mathrm{M} 31$, but this Paper demonstrates that such an effort is both underway and entirely straightforward.

AT 2016dah (also referred to as ASASSN-16hf and iPTF 16bqy) was discovered on 2016 July 12 by the intermediate Palomar Transient Factory (iPTF; Chinetti et al. 2016a), and independently two days later by the All Sky Automated Survey for Supernovae (ASAS-SN; Nicolas et al. 2016) survey. The reported position of AT 2016dah was $\alpha=0^{\mathrm{h}} 44^{\mathrm{m}} 41^{\mathrm{s}} .5, \delta=+40^{\circ} 8^{\prime} 35^{\prime \prime} .9$ (J2000), placing the system $1^{\circ} 7^{\prime} 32^{\prime \prime}$ south and $0^{\circ} 21^{\prime} 56^{\prime \prime}$ east of the centre of M 31 .

AT 2017fyp (aka ATLAS17jgy and Gaia17cgm) was discovered on 2017 August 7 by the Asteroid Terrestrial-impact Last Alert System (ATLAS; Tonry et al. 2017). AT 2017fyp is located at $\alpha=0^{\mathrm{h}} 45^{\mathrm{m}} 25^{\mathrm{s}} .490, \delta=+39^{\circ} 50^{\prime} 52^{\prime \prime} .34(\mathrm{~J} 2000), 1^{\circ} 25^{\prime} 15^{\prime \prime}$ south and $0^{\circ} 30^{\prime} 11^{\prime \prime}$ east of the centre of M31. The positions of AT 2016dah and AT 2017fyp with respect to M31 and its spectroscopically confirmed nova population (see Shafter et al. 2011; Ransome et al. in preparation) are illustrated in Fig. 1.

In this paper, we present follow-up observations of these novae located in the outer suburbs of M 31 and discuss the significance of their location within the M31 GSS. In Section 2, we describe the observations of the novae. In Section 3, we go on to describe the results of the photometric, spectroscopic, and X-ray data analysis. Then in Section 4, we explore the association of AT 2016dah and AT 2017fyp with the M31 GSS. Finally, we discuss our findings in Section 5, before summarizing our conclusions in Section 6 Throughout this paper, all quoted uncertainties are to $1 \sigma$ and all lower or upper limits are evaluated at $3 \sigma$, unless otherwise stated. 


\section{OBSERVATIONS}

\subsection{AT 2016dah}

Photometric observations of AT 2016dah were obtained using the $2.0 \mathrm{~m}$ fully robotic Liverpool Telescope (LT; Steele et al. 2004), La Palma, and the 48 arcsec Samuel Oschin telescope (P48) at Palomar. LT imaging was taken using the IO:O CCD camera ${ }^{1}$ (Smith \& Steele 2017) through $u^{\prime} B V r^{\prime} i^{\prime}$ filters, while P48 images were obtained using the CFH12K CCD mosaic camera through an $r^{\prime}$ filter. Additional photometric data were also obtained via the All-Sky Automated Survey for Supernovae (ASAS-SN; Shappee et al. 2014; Kochanek et al. 2017) Sky Patrol. ${ }^{2}$

The LT photometric data were reduced using tools within the IRAF environment (Tody 1993), and aperture photometry was performed using the QPHOT task. The LT data were calibrated against 81 stars in the field using photometry from Pan-STARRS (DR1; Chambers et al. 2016), these sources each had Pan-STARRS griz magnitudes in the range $14<m<19$ (with catalogue uncertainties $\Delta m \leq 0.1$ ). The $u^{\prime}$-band data were calibrated using a subset of 34 of those field stars that contained photometry in the range $14<u^{\prime}$ $<19\left(\Delta u^{\prime} \leq 0.1\right)$ from data release \#12 of the Sloan Digital Sky Survey (SDSS; Alam et al. 2015). The photometry of the standards was converted from Sloan to Johnson magnitudes, where required, using the appropriate transformations from Jester et al. (2005). The subsequent LT photometry is reproduced in Table S3 and information about the secondary standards is presented in Table S1. A full overview of the photometric process employed is given in Darnley et al. (2007, 2016). The P48 (iPTF) data were obtained directly from that survey's near real-time discovery pipeline (Cao, Nugent \& Kasliwal 2016; Masci et al. 2017).

Spectroscopic observations were obtained using the SPRAT (Piascik et al. 2014) instrument on the LT operating in the blueoptimized mode. A slit width of 1 ." 8 was used, resulting in a spectral resolution of $\sim 20 \AA$, or a velocity resolution of $\sim 1000 \mathrm{~km} \mathrm{~s}^{-1}$. A $\log$ of the spectroscopic observations is provided in Table 1 .

Initial reduction of the LT spectra, which includes bias subtraction, flat-field correction, and sky subtraction, up to point of wavelength calibration, was performed using the SPRAT data reduction pipeline (see Barnsley, Smith \& Steele 2012; Piascik et al. 2014). To perform relative flux calibration, we utilized 77 observations of the spectrophotometric standard star Hilt 102 (Stone 1977), taken on photometric nights between 2017 June 19 and 2017 December $31,{ }^{3}$ to construct a master sensitivity function. As the sensitivity function was constructed from data collected almost a year post-eruption, the absolute flux calibration was modified by comparison between bandpass spectrophotometry and the LT or iPTF $r^{\prime}$-band data. As such we estimate that the flux uncertainty of the AT 2016dah data is between 10 and 15 per cent.

A Neil Gehrels Swift Observatory (Gehrels et al. 2004) target of opportunity (ToO) request was approved shortly after spectroscopic confirmation of AT 2016dah (Target ID: 34619). Beginning 6.6 d post-discovery, 11 approximately weekly $S$ wift visits were utilized each with a target exposure time of $2 \mathrm{ks}$. The Swift UV/optical telescope (UVOT; Roming et al. 2005) was employed using the uvw1 filter ( 2600 $⿱$ ) . The Swift X-ray Telescope (XRT; Burrows et al. 2005) was also deployed, in the photon counting (PC) mode.

\footnotetext{
${ }^{1}$ http://telescope.livjm.ac.uk/TelInst/Inst/IOO

${ }^{2} \mathrm{https}$ ://asas-sn.osu.edu

${ }^{3}$ Prior to these dates, standards were not routinely taken by SPRAT.
}

Table 1. Summary of all spectroscopic observations of AT 2016dah and AT 2017fyp with the SPRAT spectrograph on the Liverpool Telescope.

\begin{tabular}{lcc}
\hline Date (UT) & $\Delta t(\mathrm{~d})$ & Exposure $(\mathrm{s})$ \\
\hline & AT 2016dah & \\
2016 Jul 14.084 & 2.124 & $2 \times 600$ \\
2016 Jul 14.135 & 2.175 & $3 \times 600$ \\
2016 Jul 15.077 & 3.117 & $3 \times 600$ \\
2016 Jul 16.107 & 4.174 & $3 \times 600$ \\
2016 Jul 19.101 & 7.141 & $3 \times 600$ \\
2016 Jul 26.135 & 14.115 & $3 \times 600$ \\
2016 Aug 03.093 & 22.133 & $3 \times 600$ \\
2016 Aug 05.109 & 24.149 & $3 \times 600$ \\
2016 Aug 09.094 & 28.134 & $2 \times 600$ \\
2016 Aug 13.113 & 32.153 & $3 \times 600$ \\
2016 Aug 22.093 & 41.133 & $3 \times 600$ \\
2016 Aug 29.050 & 48.090 & $5 \times 600$ \\
2016 Sep 13.127 & 63.167 & $2 \times 600$ \\
& AT 2017fyp & $3 \times 600$ \\
2017 Aug 11.097 & 5.017 & $3 \times 600$ \\
2017 Aug 13.018 & 6.938 & $3 \times 600$ \\
2017 Aug 15.081 & 9.001 & $3 \times 600$ \\
2017 Aug 17.132 & 11.052 & $3 \times 600$ \\
2017 Aug 20.117 & 14.037 & $3 \times 600$ \\
2017 Aug 26.105 & 20.025 & $3 \times 600$ \\
2017 Sep 01.040a & 25.960 & $3 \times 900$ \\
2017 Sep 16.045 & 40.965 & $3 \times 900$ \\
2017 Sep 30.126 & 55.046 & $3 \times 900$ \\
2017 Oct 18.939 & 73.859 & \\
2017 Nov 12.912 & 98.832 & \\
\hline
\end{tabular}

${ }^{a}$ The night of 2017 August 31 was non-photometric and this spectrum was collected through cloud. We have not included it in the analysis due to the poor signal-to-noise ratio.

The Swift data were processed using the HEASOFT (v6.26.1 for UVOT; v6.22 for XRT) software and the corresponding calibration files. UVOT magnitudes were calculated using the uvotsource tool, with a standard 5 arcsec radius circular extraction region for the source, and a 46 arcsec radius circular aperture offset from, but close to $\left(\alpha=0^{\mathrm{h}} 44^{\mathrm{m}} 43^{\mathrm{s}}, \delta=40^{\circ} 09^{\prime} 34^{\prime \prime}\right)$, the source used to estimate the background. The UV emission had faded below detectability by the end of September. The XRT data were analysed using the freely available on-line tool ${ }^{4}$ (Evans et al. 2009). No X-ray source corresponding to AT 2016dah was detected at any time (also see Chinetti et al. 2016b). Results from the UVOT and XRT analysis are presented in Table 2.

For each Swift XRT observation, we have also estimated the upper luminosity limit of the source $(0.3-10 \mathrm{keV})$. Here, we assumed a distance to M 31 of $d=752 \pm 17 \mathrm{kpc}$ (Freedman et al. 2001), an estimate of the Galactic neutral atomic $H$ density $n_{\mathrm{H}}=5.32 \times 10^{20} \mathrm{~cm}^{-2}$ (HI4PI Collaboration 2016), and a typical (for X-ray detected M31 novae) blackbody temperature of $k T=$ $50 \mathrm{eV}$ (Henze et al. 2014). Luminosity limits were estimated using the web interface to PIMMS (v4.10b) ${ }^{5}$ and are recorded in Table 2.

\subsection{AT 2017fyp}

The LT photometric data for AT 2017fyp were reduced in a similar manner as those for AT 2016dah. Here, the LT data were calibrated against 42 stars in the field using photometry from SDSS DR12,

\footnotetext{
${ }^{4}$ https://www.swift.ac.uk/user_objects

${ }^{5}$ https://heasarc.gsfc.nasa.gov/cgi-bin/Tools/w3pimms/w3pimms.pl
} 
Table 2. Neil Gehrels Swift Observatory XRT and UVOT observations of AT 2016dah and AT 2017fyp.

\begin{tabular}{|c|c|c|c|c|c|c|c|c|}
\hline \multirow{2}{*}{$\begin{array}{l}\text { Date } \\
\text { (UT) }\end{array}$} & \multirow{2}{*}{$\begin{array}{l}\Delta t \\
\text { (d) }\end{array}$} & \multicolumn{2}{|c|}{ MJD $57000+$} & \multirow{2}{*}{$\begin{array}{l}\text { Exposure } \\
\text { time }^{\text {b }}(\mathrm{s})\end{array}$} & \multicolumn{2}{|c|}{ XRT (0.3-10 keV) } & \multirow{2}{*}{$\begin{array}{l}\text { UVOT uvw1 } \\
\qquad(\mathrm{mag})^{b}\end{array}$} & \multirow[t]{2}{*}{ Obs. ID } \\
\hline & & Start & End & & $\left(\right.$ counts s $\left.^{-1}\right)$ & $L\left(10^{36} \mathrm{erg} \mathrm{s}^{-1}\right)$ & & \\
\hline \multicolumn{9}{|c|}{ AT 2016dah } \\
\hline 2016 Jul 19.377 & 7.417 & 588.008 & 588.746 & 2041.8 & $<0.008$ & $<3.6$ & $17.12 \pm 0.03$ & 00034619002 \\
\hline 2016 Jul 26.278 & 14.318 & 595.237 & 595.319 & 2044.1 & $<0.005$ & $<2.3$ & $18.00 \pm 0.05$ & 00034619003 \\
\hline 2016 Aug 03.621 & 22.661 & 603.356 & 603.887 & 1183.4 & $<0.032$ & $<14.4$ & $18.25 \pm 0.07$ & 00034619004 \\
\hline 2016 Aug 11.903 & 30.943 & 611.864 & 611.941 & 1847.0 & $<0.006$ & $<2.7$ & $19.11 \pm 0.09$ & 00034619005 \\
\hline 2016 Aug 20.677 & 39.717 & 620.642 & 620.712 & 1181.8 & $<0.007$ & $<3.2$ & $19.36 \pm 0.13$ & 00034619006 \\
\hline 2016 Sep 04.695 & 54.735 & 635.654 & 635.736 & 2066.8 & $<0.004$ & $<1.8$ & $20.26 \pm 0.19$ & 00034619007 \\
\hline 2016 Sep 12.799 & 62.839 & 643.768 & 643.830 & 1281.0 & $<0.019$ & $<8.5$ & $20.49 \pm 0.29$ & 00034619008 \\
\hline 2016 Sep 20.773 & 70.813 & 651.732 & 651.814 & 1902.2 & $<0.007$ & $<3.2$ & $20.50 \pm 0.23$ & 00034619009 \\
\hline 2016 Sep 29.772 & 79.812 & 660.767 & 660.778 & 954.5 & $<0.044$ & $<19.8$ & $\ldots{ }^{\mathrm{c}}$ & 00034619011 \\
\hline 2016 Oct 02.889 & 82.929 & 663.888 & 663.889 & 116.3 & $<0.157$ & $<70.6$ & $>19.2$ & 00034619012 \\
\hline 2016 Oct 06.714 & 86.754 & 667.675 & 667.752 & 1966.5 & $<0.005$ & $<2.3$ & $(21.09 \pm 0.38)^{\mathrm{d}}$ & 00034619013 \\
\hline \multicolumn{9}{|c|}{ AT 2017fyp } \\
\hline 2017 Aug 24.802 & 18.722 & 989.702 & 989.901 & 1836.0 & $<0.015$ & $<6.2$ & $16.40 \pm 0.03$ & 00010239001 \\
\hline 2017 Sep 07.101 & 32.021 & 1003.002 & 1003.200 & 451.2 & $<0.021$ & $<8.7$ & $17.14 \pm 0.06$ & 00010239002 \\
\hline 2017 Sep 14.504 & 39.424 & 1010.368 & 1010.641 & 1401.0 & $<0.020$ & $<8.2$ & $17.73 \pm 0.05$ & 00010239003 \\
\hline 2017 Oct 18.279 & 73.199 & 1044.116 & 1044.441 & 3695.4 & $<0.003$ & $<1.2$ & $18.65 \pm 0.05$ & 00010239004 \\
\hline 2017 Nov 18.190 & 104.110 & 1075.115 & 1075.264 & 4049.2 & $<0.002$ & $<0.8$ & $19.36 \pm 0.08$ & 00010239005 \\
\hline 2017 Dec 18.593 & 134.513 & 1105.224 & 1105.962 & 3149.9 & $<0.004$ & $<1.6$ & $20.43 \pm 0.17$ & 00010239006 \\
\hline 2018 Jan 18.776 & 165.696 & 1136.773 & 1136.778 & 425.9 & $<0.051$ & $<21.0$ & $>20.0$ & 00010239007 \\
\hline 2018 Jan 20.492 & 167.412 & 1138.490 & 1138.494 & 322.7 & $<0.025$ & $<10.3$ & $>19.6$ & 00010239008 \\
\hline 2018 Jan 21.961 & 168.881 & 1139.760 & 1140.162 & 1153.8 & $<0.011$ & $<4.5$ & $>20.5$ & 00010239009 \\
\hline 2018 Jan 23.920 & 170.840 & 1141.887 & 1141.953 & 2209.9 & $<0.006$ & $<2.5$ & $>20.9$ & 00010239010 \\
\hline 2018 Jan $18-24^{e}$ & 168.284 & 1136.773 & 1141.953 & 3822.3 & $<0.003$ & $<1.2$ & $>21.2$ & $\ldots 007-010$ \\
\hline
\end{tabular}

${ }^{a}$ Here, the exposure time refers specifically to UVOT.

${ }^{b}$ UVOT magnitudes are reported in the Vega system, we quote the random statistical uncertainties, calibration systematic uncertainties are 0.03 mag.

${ }^{c}$ No useable data due to loss of star tracker lock.

${ }^{d}$ Source detection significance at the nova position was at $2.9 \sigma$.

${ }^{e}$ Here, we combine the final four Swift observations as they were taken within a one week period.

these sources each had Sloan $u^{\prime} g^{\prime} r^{\prime} i^{\prime} z^{\prime}$ magnitudes in the range $14<$ $m<19(\Delta m \leq 0.1)$. The subsequent LT photometry is reproduced in Table $\mathrm{S} 4$ and information about the secondary standards is presented in Table S2.

The Las Cumbres Observatory (LCO; Brown et al. 2013) 2.0-m telescope at Haleakala (formally the Faulkes Telescope North) was used to collect four additional epochs of $B V r^{\prime} i^{\prime}$ photometry. These data were taken using the Spectral camera ${ }^{6}$ and were reduced and analysed in an identical manner to the LT imaging data. The LCO photometry is also recorded within Table S4.

Additional photometric data for AT 2017fyp, limited to the pre-discovery and discovery photometry, were obtained from the ATLAS survey (Tonry et al. 2017). Three Gaia photometry points are also available. ${ }^{7}$

The LT SPRAT spectroscopic data were reduced in an identical manner to those for AT 2016dah, and we employed the same sensitivity function to produce relative flux calibrated spectra. However, for these data, no discernible improvement in the absolute flux calibration was found by using bandpass spectrophotometry, in part this was due to the lower photometric cadence. We estimate the flux uncertainty of the AT 2017fyp data is between 15 and 20 per cent.

A Swift ToO programme was also rapidly approved postdiscovery for AT 2017fyp (Target ID: 10239) and a series of 10 observations commenced two weeks post-discovery. These Swift XRT and UVOT data were reduced in an identical fashion to those

\footnotetext{
${ }^{6}$ https://lco.global/observatory/instruments/spectral

${ }^{7}$ http://gsaweb.ast.cam.ac.uk/alerts/alert/Gaia17cgm
}

for AT 2016dah. For the UVOT photometry, a circular background estimation region of radius 67 arcsec as positioned at $\alpha=0^{\mathrm{h}} 45^{\mathrm{m}} 34^{\mathrm{s}}$, $\delta=39^{\circ} 49^{\prime} 25^{\prime \prime}$. Like AT 2016dah, no X-ray source was detected, here upper luminosity limits were derived assuming an average Galactic column of $n_{\mathrm{H}}=4.73 \times 10^{20} \mathrm{~cm}^{-2}$. The AT 2017fyp swift results are also reported in Table 2 . The final four Swift visits were obtained within a single week, combining them does not yield either an XRT or UVOT detection at that time.

\subsection{Archival data}

To enable a search for the progenitor systems of AT 2016dah and AT 2017fyp, i.e. the quiescent novae, we utilized a number of archival observations. The positions of both AT 2016dah and AT 2017fyp are located within Field 7 of the Canada-FranceHawaii Telescope (CFHT) survey of the M31 GSS McConnachie et al. (2003, also see Sections 4 and 5.6). These data were taken using the $\mathrm{CFH} 12 \mathrm{~K}$ instrument, a 12, $288 \times 8,192$ pixel CCD mosaic camera (Cuillandre et al. 2000) and consist of a pair of $3 \times 545 \mathrm{~s}$ exposures through Mould $V$ - and $I$-band filters. The CFHT data were collected on 2001 September 13, 15 yr before the eruptions. AT 2016dah lies within chip02 of Field 7, whereas AT 2017fyp can be found within chip09. As the field of view of each of the CFH12K chips is roughly similar to the LT field, these data were processed in a similar fashion to the LT data, including stacking of each set of three images, and photometry was performed as described above. We also utilized the Galaxy Evolution Explorer (GALEX; Martin et al. 2005) data archive to extend the search to the near- and far-UV.

A search for potential progenitor/quiescent systems was performed following the procedure set out in Bode et al. (2009), and 


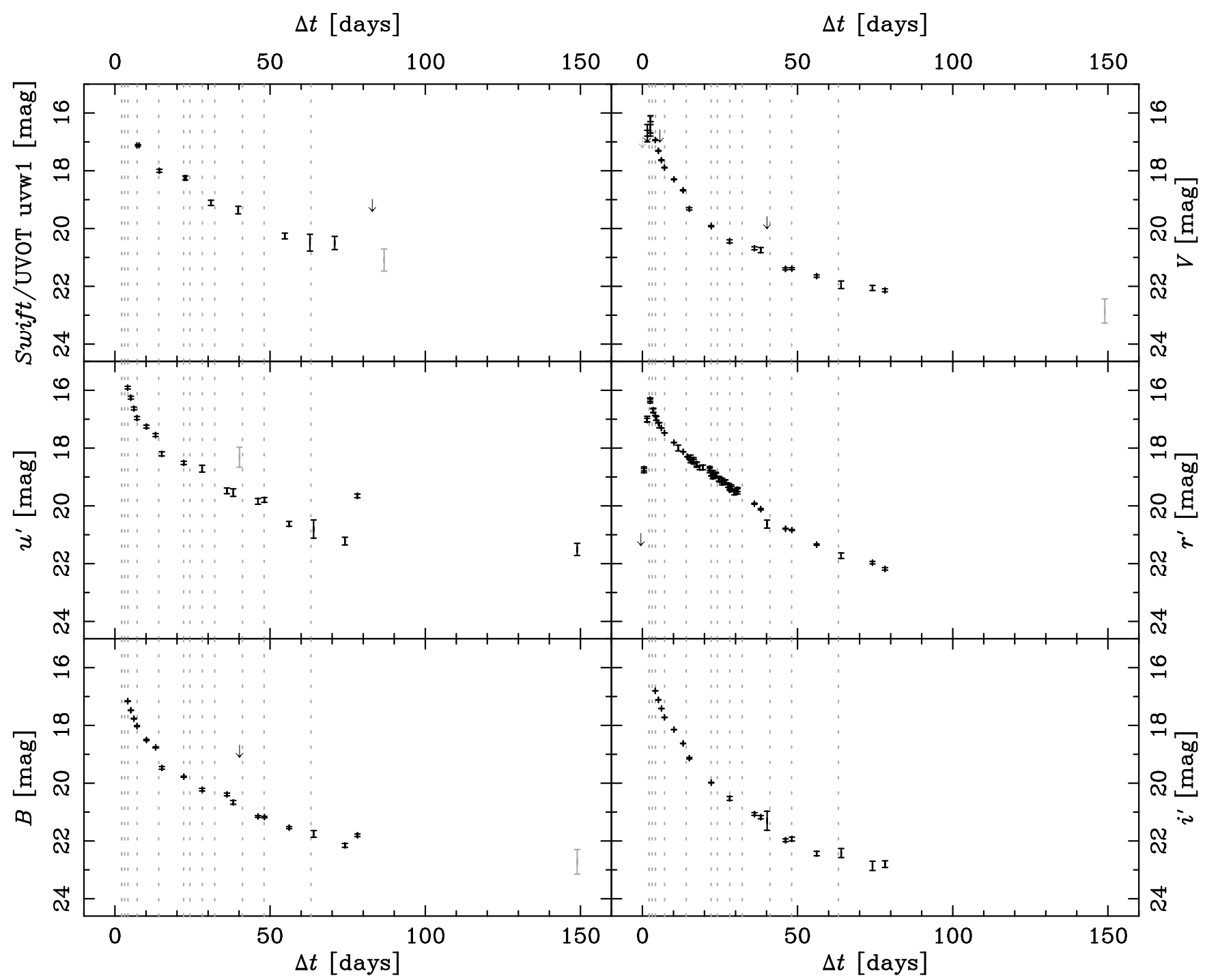

Figure 2. The near-UV - optical light curve of nova AT 2016dah. The epochs of spectroscopy are also indicated by the vertical grey-dotted lines. Grey data points indicate detections with significance $2<\sigma \leq 3$.

then further refined by Williams et al. (2014) and more recently Healy et al. (2019). Astrometric solutions were computed between each $V$ - and $i^{\prime}$-band LT observation and the corresponding CFHT $V$ - and $I$-band stack, and the GALEX data, to define the progenitor search region with those data.

\section{RESULTS}

\subsection{Time of eruption}

Although the last pre-discovery observation of AT 2016dah was taken in the $V$ band by ASAS-SN on 2016 July 11.52 UT, this observation was not of sufficient depth to rule out an eruption. Therefore, we assume that the eruption occurred between the last iPTF non-detection $(R>21.171)$ on July 11.48 and the iPTF detection $(R=18.78 \pm 0.07)$ on July 12.44 . For the purpose of our analysis, we take the time of eruption to be 2016 July $11.96 \pm 0.48$ UT (MJD: $57580.96 \pm 0.48$ ).

AT 2017fyp was observed by ATLAS on 2017 August 04.603 UT; no source was detected down to a limit of 19.28 mag (through the
ATLAS orange filter). We again assume that the eruption occurred at some point between that observation and the discovery observation, also by ATLAS, on August 07.553. Therefore we take the time of eruption of AT 2017fyp to be 2017 Aug $6.08 \pm 1.48$ UT (MJD: $57971.08 \pm 1.48)$.

\subsection{Reddening}

The general Galactic reddening towards M 31 was determined as $E(B-V)=0.1$ (Stark et al. 1992). Schlegel, Finkbeiner \& Davis (1998) find $E(B-V)=0.06$ and 0.05 around the positions of AT 2016dah and AT 2017fyp, respectively. Converting the Galactic $n_{\mathrm{H}}$ estimates (see Section 2) to reddening (using equation 1 from Güver \& Özel 2009, and assuming $\left.R_{V}=3.1\right)$ we find $E(B-V)=$ 0.08 and 0.07 for AT 2016dah and AT 2017fyp, respectively. There are no reliable reddening markers available for nova photometry, and there are none present in our obtained spectra with which we could independently constrain the reddening towards either system. Some authors have used the Balmer decrement to estimate the reddening for particular novae, however others (see for e.g. for 

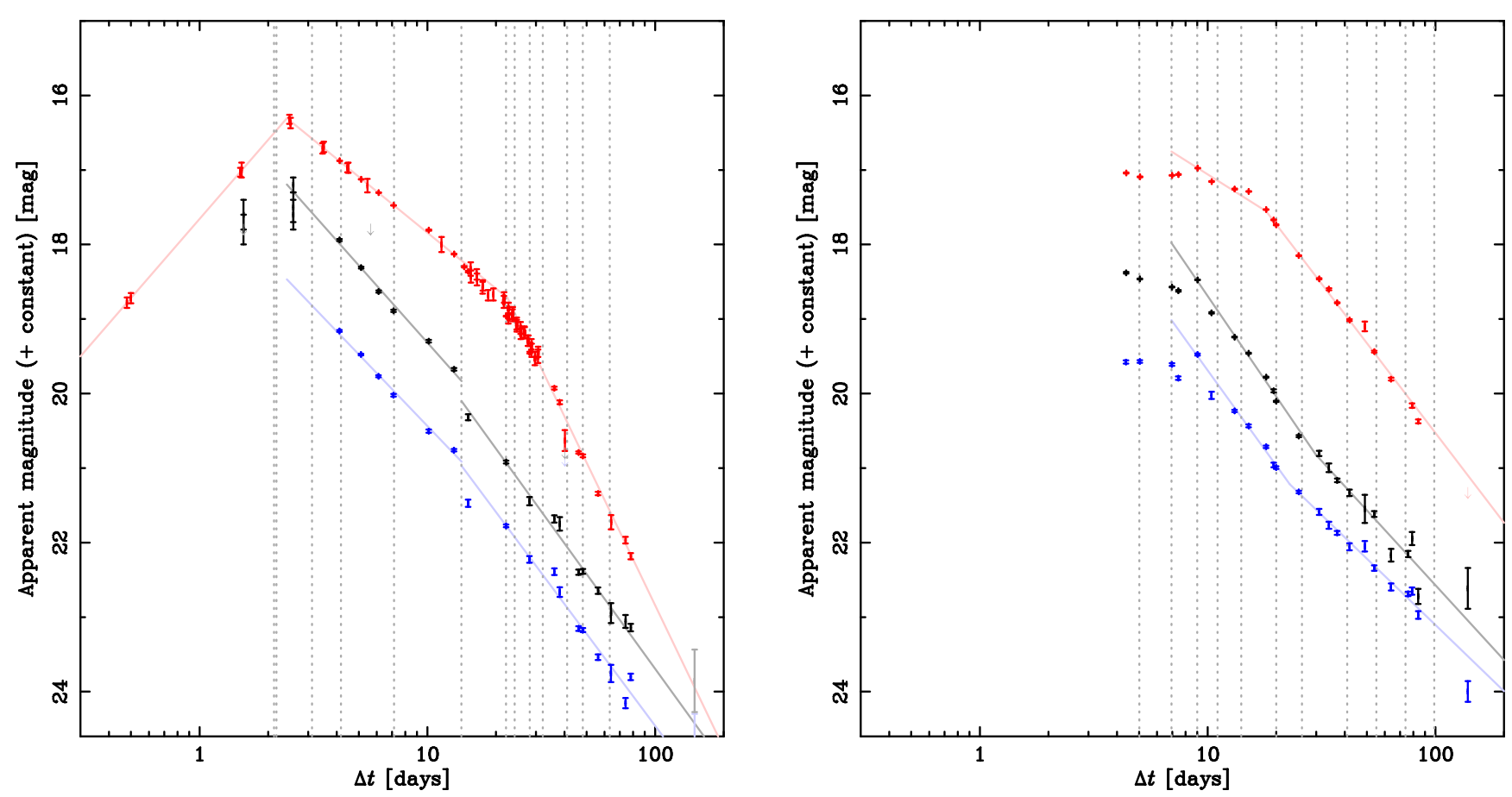

Figure 3. Light curve of AT 2016dah (left) and AT 2017fyp (right) displayed with logarithmic time axes. The $r^{\prime}$-band light curve is shown in red (top), $V$ band in black (middle; all magnitudes shifted by +1 ), and $B$ band in blue (bottom; shifted by +2 ). The broken power-law fits to these data $\left(F \propto t^{\alpha}\right)$ are discussed in the text. The vertical dotted lines indicate spectroscopic epochs.

a recent discussion Williams, Darnley \& Henze 2017) have shown this to be unreliable. As such, we will assume a reddening of $E(B$ $-V)=0.1$ towards both novae. ${ }^{8}$ Given that both novae are located far from the M31 disc, we assume that there is negligible extinction contribution beyond the confines of the Milky Way. All spectra have been dereddened using this value, and all subsequent discussions of the spectra refer to analysis of the dereddened observations.

\subsection{Photometric evolution}

\subsubsection{AT 2016dah}

The combined ASAS-SN, iPTF, LT, and Swift UVOT light curve of the eruption of AT 2016dah are shown in Fig. 2. These light curves reveal a nova that was detected on the rise (still relatively uncommon for extragalactic novae, particularly those beyond the Magellanic Clouds), that in general follows a rapid and smooth decline through around six magnitudes (before it became undetectable). The Strope, Schaefer \& Henden (2010) light curve scheme would classify AT 2016dah as a smooth or 'S'-type nova.

In Fig. 3, we present the $r^{\prime}$-band light curve (red) of AT 2016dah, plotted on a logarithmic time axis. The three straight-line fits to these data, demonstrate that the $r^{\prime}$-band evolution follows three broken power laws (between flux and time; $F \propto t^{\alpha}$ ). The post-maximum break occurs at around $22.5 \mathrm{~d}$ post-eruption, before the break the slope is $\alpha=-1.00 \pm 0.01$; post-break, $\alpha=-2.55 \pm 0.06$. We note

\footnotetext{
${ }^{8}$ The recurrent nova M31N 2008-12a lies in front of the bulk dust and gas in the M 31 structural model proposed by Dalcanton et al. (2015). Darnley et al. (2017a) directly measured the reddening towards M31N 2008-12a to be $E(B-V)=0.10 \pm 0.03$, which is consistent with the Stark et al. (1992), Schlegel et al. (1998), and HI4PI Collaboration et al. (2016) determinations.
}

that neither value is consistent with those determined by Hachisu \& Kato (2006). The slope of the $r^{\prime}$-band light cure before maximum light is $\alpha=1.41 \pm 0.01$.

Fig. 3 also shows the $V$-band (black) and $B$-band (blue) light curves. While much less densely sampled than the $r^{\prime}$-band data, these light curves follow a similar form, with one marked difference. There is an apparent 'discontinuity' in the light curves at around day 14 (a spectrum was also taken at this time, see Section 3.4.1), where there is a 'sudden' drop in the brightness by just over half a magnitude. This drop also appears in the $u^{\prime}$ band and there is a non-conclusive hint of such an occurrence in the Swift UVOT data, but is not present in the two reddest filters. The $V$ - and $B$-band data from maximum light until this drop are consistent with power law of indices of $\alpha_{V}=-1.38 \pm 0.06$ and $\alpha_{B}=-1.30 \pm 0.05$, respectively, i.e. they are consistent with each other, but not with the $r^{\prime}$ band decline during this phase. Post-drop, the $V$ and $B$ band declines remain consistent with indices of $\alpha_{V}=-1.69 \pm 0.05$ and $\alpha_{B}=-1.65 \pm 0.07$, respectively. Both these later time $V$ and $B$ band declines are consistent with the power-law slopes expected during this period between $\sim 2$ and $\sim 6$ mag below peak $(\alpha=-1.75$; Hachisu \& Kato 2006).

For the purpose of this analysis, we will assume that maximum light occurred at the time of the brightest $r^{\prime}$-band observation, $r^{\prime}=16.32 \pm 0.06,2.48 \mathrm{~d}$ post-eruption. A pair of spectra taken $0.33 \mathrm{~d}$ earlier (see Section 3.4.1) are consistent with pre-maximum evolution, a spectrum taken at $\Delta t=3.117$ shows the nova in a post-maximum state. As such, we can use the power-law fits to derive the following estimates of the $t_{2}$ and $t_{3}$ decline times (the time taken to decay by two and three magnitudes, respectively, from peak): $t_{2}\left(r^{\prime}\right)=13.3_{-0.3}^{+0.6} \mathrm{~d}$ (a decay rate of $\sim 0.15 \mathrm{mag} \mathrm{d}^{-1}$ ); $t_{3}\left(r^{\prime}\right)=26 \pm 2 \mathrm{~d}$ and occurs $\sim 6 \mathrm{~d}$ post-break. Such decline times would class AT 2016dah as a fast nova (Payne-Gaposchkin 1964). 


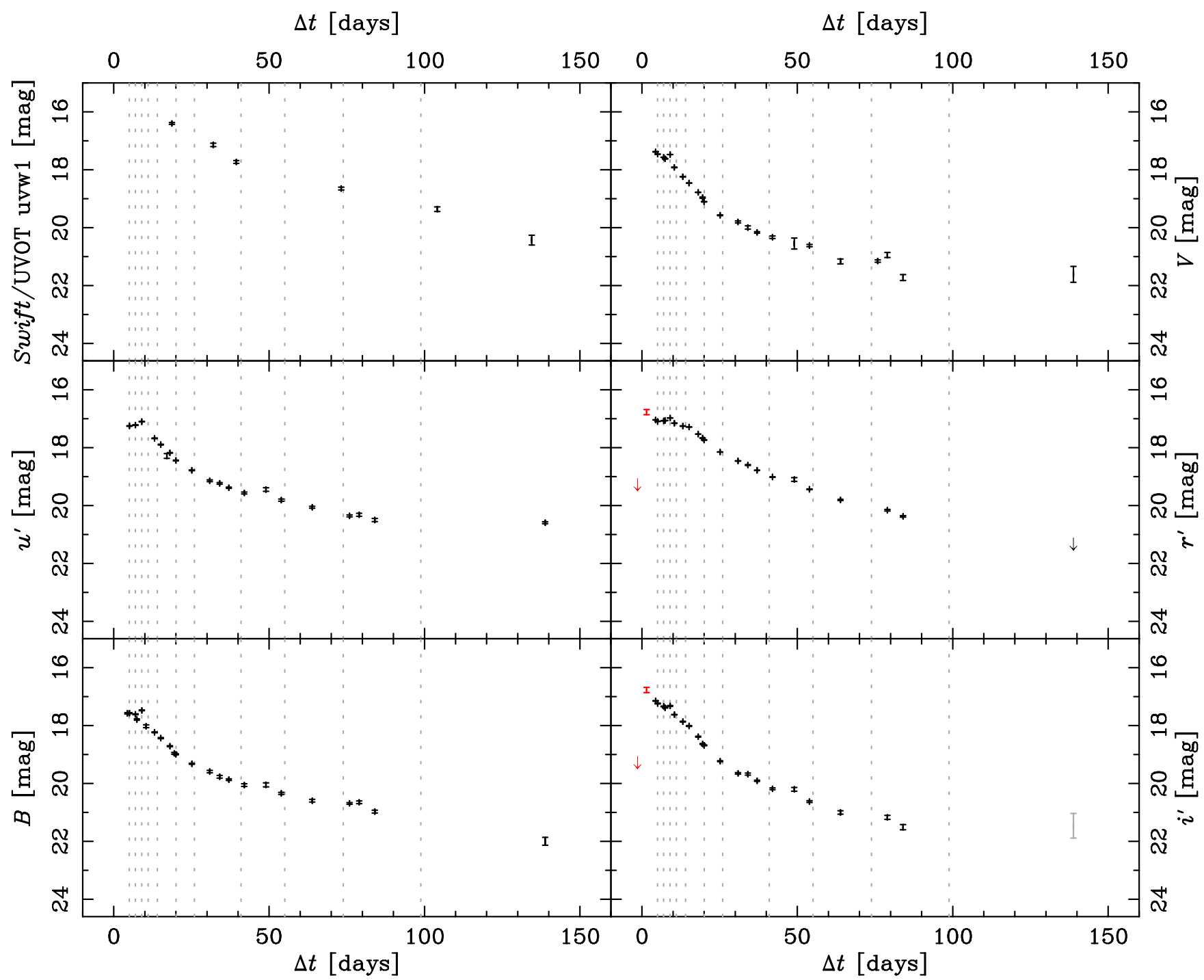

Figure 4. The near-UV-optical light curve of nova AT 2017fyp. The epochs of spectroscopy are also indicated by the vertical grey-dotted lines. Grey data points indicate detections with significance $2<\sigma \leq 3$. The red data points included in the $r^{\prime}$ and $i^{\prime}$ indicate the ATLAS orange filter pre-discovery upper limit and discovery photometry; the orange filter has a wide bandpass that covers $r^{\prime}+i^{\prime}$. The final four Swift data points $(\Delta t>50 \mathrm{~d}$; see Table 2) are not shown, all four are upper limits consistent with the expected late-decline of the eruption.

For the $V$ and $B$ bands we find, $t_{2}(V)=7 \pm 1 \mathrm{~d}, t_{2}(B)=8 \pm 1 \mathrm{~d}$, $t_{3}(V)=13 \pm 1 \mathrm{~d}$, and $t_{3}(B)=16_{-2}^{+3} \mathrm{~d}$. These values are smaller than those computed using the $r^{\prime}$-band data, whose decline is slowed by the persistence of the $\mathrm{H} \alpha$ emission line. The $V$ - and $B$-band values classify AT 2016dah as a very fast nova, the $V$-band value for $t_{3}$ is consistent with the epoch of the light curve 'drop', the uncertainty on the $B$-band $t_{3}$ is larger due to the lack of observations at maximum light, but it is formally also consistent with the drop.

The light curve of AT 2016dah was followed for around $150 \mathrm{~d}$ post-eruption, at which point the nova was detected $\sim 6$ mag below peak in the $u^{\prime}$ band, and was marginally detected (sub $3 \sigma$ ) with a similar amplitude in the $V$ and $B$ bands.

\subsubsection{AT 2017fyp}

In Fig. 4, we present the ATLAS, LCO, LT, and Swift/UVOT photometric light curves of AT 2017fyp. At first inspection, the evolution of AT 2017fyp is clearly slower than that of AT 2016dah, and it is around $\sim 1 \mathrm{mag}$ fainter at peak. There is no coverage of the rise, and maximum light may have been missed, but the light curve appears to have a short flat-top peak lasting around $\sim 5 \mathrm{~d}$. Following this flat period, the nova enters a rapid decline until a clear break in the light curve at $\sim 25 \mathrm{~d}$ post-eruption where the decline slows. AT 2017 fyp was followed for $\sim 140 \mathrm{~d}$ post-eruption through a decay of 4-5 mag, after which it remained detected in the bluer bands (and was marginally detected, $<3 \sigma$, in the $i^{\prime}$ band). Given the flat topped nature, and power law like decline (see below), AT 2017fyp may be best classed as a flat topped or 'F'type nova. A slight increase in the luminosity of the nova during this maximum light 'plateau' phase is evident, particularly to the blue.

As with AT 2016dah, we present the $B V r^{\prime}$-band light curves of AT 2017fyp in Fig. 3, where they are plotted against a logarithmic time axis. Here, the flat topped nature is evident, as is the brokenpower law decline, with that break in the opposite direction in 
the $V$ and $B$ bands to that of AT 2016dah (steep to shallow, for AT 2017fyp, versus shallow to steep for AT 2016dah). Here, the $r^{\prime}$ band behaviour is again probably driven by the $\mathrm{H} \alpha$ emission line evolution.

The initial indices of the $B V r^{\prime}$-band power-law declines are $\alpha=$ $-1.69 \pm 0.07,-1.79 \pm 0.06$, and $-0.77 \pm 0.08$, respectively. Here the $V$ - and $B$-band slopes are consistent with those determined by Hachisu \& Kato (2006), but over an earlier part of the light-curve development (as measured by decline from peak) as suggested by those authors. The later portion of the decline is consistent with power-law indices of $\alpha=-1.18 \pm 0.04,-1.35 \pm 0.05$, and $-1.60 \pm 0.04$, for the $B V r^{\prime}$-bands, respectively.

With novae with flat topped or cusp-like (' $\mathrm{C}$ '-type) behaviour around peak, it is always a bone of contention how one should determine the epoch of maximum light, and hence how to reliably estimate the decline times. For this analysis, we will assume that maximum light occurred at some point during the $\sim 5 \mathrm{~d}$ flat top (but no later than $\Delta t \approx 9 \mathrm{~d}$ post-eruption), and that the decline time range is determined by the power-law decline and the width of the flat top. As such, we estimate the following decline times: $32 \lesssim$ $t_{2}\left(r^{\prime}\right) \lesssim 37 \mathrm{~d}, 16 \lesssim t_{2}(V) \lesssim 21 \mathrm{~d}, 20 \lesssim t_{2}(B) \lesssim 25 \mathrm{~d}, 63 \lesssim t_{3}\left(r^{\prime}\right) \lesssim$ $68 \mathrm{~d}, 38 \lesssim t_{3}(V) \lesssim 43 \mathrm{~d}$, and $53 \lesssim t_{3}(B) \lesssim 58 \mathrm{~d}$. The $V$ and $B$ band decline times would class AT 2017fyp as a fast nova, whereas the slower $r^{\prime}$-band times would class this eruption as moderately fast, again the influence of the $\mathrm{H} \alpha$ emission line will have impacted the $r^{\prime}$-band estimates.

\subsection{Spectroscopic evolution}

\subsubsection{AT 2016dah}

A series of 13 spectra of AT 2016dah were obtained by the SPRAT instrument on the LT. The first being captured at 2016 July $14.084 \mathrm{UT}$, just $2.12 \mathrm{~d}$ post-eruption. That spectrum, and a second obtained $0.05 \mathrm{~d}$ later (see the black and red spectra - the two most luminous - in the top panel of Fig. 5), is both consistent with the early optically thick 'fireball' phase of a nova eruption (Chinetti et al. 2016a). These spectra were observed $\sim 0.33 \mathrm{~d}$ before $r^{\prime}$-band maximum light (see Fig. 2). This pair of spectra exhibit weak Balmer emission with the $\mathrm{H} \alpha-\mathrm{H} \delta$ line profiles all containing PCygni absorption troughs to the blue. The $\mathrm{H} \alpha$ absorption minimum is consistent with a velocity of $-1200 \pm 200 \mathrm{~km} \mathrm{~s}^{-1}$, with respect to the mean emission peak (see below). Whereas the terminal velocity of the $\mathrm{H} \alpha$ PCygni is at $2300 \pm 400 \mathrm{~km} \mathrm{~s}^{-1}$ blueward of the mean peak. These spectra display a blue continuum with relatively few other features. The continuum may be punctuated by blueshifted absorptions from Fe II multiplets 42 and 74. At this stage emission from O I (1) $7774 \AA$ is already present. A fit to the continua of this pair of spectra shows that they are consistent with the form of a blackbody with effective temperature $\approx 10000 \mathrm{~K}$.

A Gaussian profile combined with a linear function was used to simultaneously fit the line flux and local continuum for the Balmer emission lines $(\mathrm{H} \alpha-\mathrm{H} \gamma$; the low signal-to-noise ratio and line blanketing, due to the low spectral resolution to the blue, rendered fits to $\mathrm{H} \delta$ uninformative). The resultant line fluxes are reproduced in Table 3. The evolution of the Balmer emission line fluxes and that of the $\mathrm{H} \alpha$ line profile is shown in the top-panel of Fig. 6. The Balmer emission peaks much later than the broad-band maximum light (which occurs at $\Delta t=2.48 \mathrm{~d}$ post-eruption). Following a slow increase in flux, the Balmer emission peaks around day 7 posteruption. After peak, the Balmer emission follows a roughly linear
Table 3. Selected emission line fluxes from the Liverpool Telescope SPRAT spectra of AT 2016dah and AT 2017fyp.

\begin{tabular}{|c|c|c|c|}
\hline \multirow[t]{2}{*}{$\Delta t(\mathrm{~d})$} & \multicolumn{3}{|c|}{$\begin{array}{c}\text { Dereddened flux } \\
{\left[\times 10^{-15} \mathrm{erg} \mathrm{cm}^{-2} \mathrm{~s}^{-1}\right]}\end{array}$} \\
\hline & $\mathrm{H} \alpha$ & $\mathrm{H} \beta$ & $\mathrm{H} \gamma$ \\
\hline \multicolumn{4}{|c|}{ AT 2016dah } \\
\hline $2.124^{\mathrm{a}}$ & $8 \pm 3$ & $16 \pm 3$ & $7 \pm 2$ \\
\hline $2.175^{\mathrm{a}}$ & $8 \pm 3$ & $17 \pm 5$ & $(5 \pm 3)$ \\
\hline 3.117 & $52 \pm 3$ & $54 \pm 5$ & $40 \pm 7$ \\
\hline 4.174 & $111 \pm 3$ & $93 \pm 6$ & $64 \pm 6$ \\
\hline 7.141 & $183 \pm 3$ & $85 \pm 4$ & $43 \pm 6$ \\
\hline 14.115 & $136 \pm 3$ & $35 \pm 3$ & $18 \pm 4$ \\
\hline 22.133 & $66 \pm 1$ & $16 \pm 1$ & $8 \pm 1$ \\
\hline 24.149 & $69 \pm 2$ & $16 \pm 1$ & $12 \pm 2$ \\
\hline 28.134 & $50 \pm 2$ & $11 \pm 2$ & $(7 \pm 4)$ \\
\hline 32.153 & $39 \pm 1$ & $10 \pm 2$ & $7 \pm 2$ \\
\hline 41.133 & $15 \pm 1$ & $3 \pm 1$ & $(3 \pm 2)$ \\
\hline 48.090 & $13 \pm 1$ & $3 \pm 1$ & $(3 \pm 1)$ \\
\hline 63.167 & $2 \pm 1^{b}$ & $\ldots$ & $\ldots$ \\
\hline \multicolumn{4}{|c|}{ AT 2017fyp } \\
\hline 5.017 & $202 \pm 7$ & $130 \pm 10$ & $60 \pm 10$ \\
\hline 6.938 & $310 \pm 10$ & $140 \pm 20$ & $75 \pm 7$ \\
\hline 9.001 & $420 \pm 20$ & $160 \pm 20$ & $90 \pm 20$ \\
\hline 11.052 & $440 \pm 20$ & $140 \pm 20$ & $78 \pm 9$ \\
\hline 14.037 & $350 \pm 20$ & $100 \pm 9$ & $49 \pm 6$ \\
\hline 20.025 & $300 \pm 10$ & $60 \pm 4$ & $31 \pm 4$ \\
\hline 40.965 & $92 \pm 5$ & $22 \pm 2$ & $17 \pm 3$ \\
\hline 55.046 & $53 \pm 3$ & $12 \pm 2$ & $14 \pm 3$ \\
\hline 73.859 & $39 \pm 2$ & $8 \pm 2$ & $14 \pm 3$ \\
\hline 98.832 & $21 \pm 1$ & $5 \pm 2$ & $6 \pm 1$ \\
\hline
\end{tabular}

$\overline{\text { Notes. All emission line fluxes and uncertainties were determined through }}$ fitting a Gaussian profile to the line in velocity space. Values in parenthesis are lines for which the flux uncertainties lie between $2 \sigma$ and $3 \sigma$.

${ }^{a}$ In these spectra the Balmer lines have P Cygni profiles, here we report the flux of the emission component.

${ }^{b}$ Formally, the flux was constrained marginally beyond $3 \sigma$.

decline (see Fig. 6). We measure the weighted average emission line centre of $\mathrm{H} \alpha$ from all 13 spectra to be at $-420 \pm 30 \mathrm{~km} \mathrm{~s}^{-1}$ (after heliocentric correction) with respect to the rest-wavelength of $\mathrm{H} \alpha$, which corresponds to a redshift of $z=(-1.4 \pm 0.1) \times$ $10^{-3}$. The weighted average FWHM of $\mathrm{H} \alpha$ across the 13 spectra is $2300 \pm 70 \mathrm{~km} \mathrm{~s}^{-1}$. Other than during the first two epochs, where the $\mathrm{H} \alpha$ profile has a PCygni form, there is not significant evolution of the FWHM through the spectral sequence. In general, the $\mathrm{H} \alpha$ profile is Gaussian-like, which is fairly typical for Fe II novae.

The following three spectra were taken, $3.12,4.17$, and $7.14 \mathrm{~d}$ post-eruption (see green, blue, and cyan, respectively, spectra in the top panel of Fig. 5) and were all collected between maximum light and $t_{2}$, with the last of this sub-set taken just over 1 mag below peak. Here, there is a clear transition from the fireball phase to the 'principle' spectrum. The continuum emission weakens across the sub-set, it is no longer blackbody like, and is shallower than that expected from a Rayleigh-Jeans tail. Throughout this stage the continuum slope is not consistent with that expected from freefree emission (see Wright \& Barlow 1975). The Balmer series has transitioned from their initial PCygni profiles to strong emission lines that contribute substantially to the total optical emission. The Fe II (42) triplet is the next strongest feature, with emission also detected from Fe II $(26,27,37,38,48,49$, and 74). Given the lack of Fe II (74) $6248 \AA$, the Fe II (74) $6148 \AA$ line is probably blended with O I $6157 \AA$. Emission from O I (1) $7774 \AA$ A remains present and 

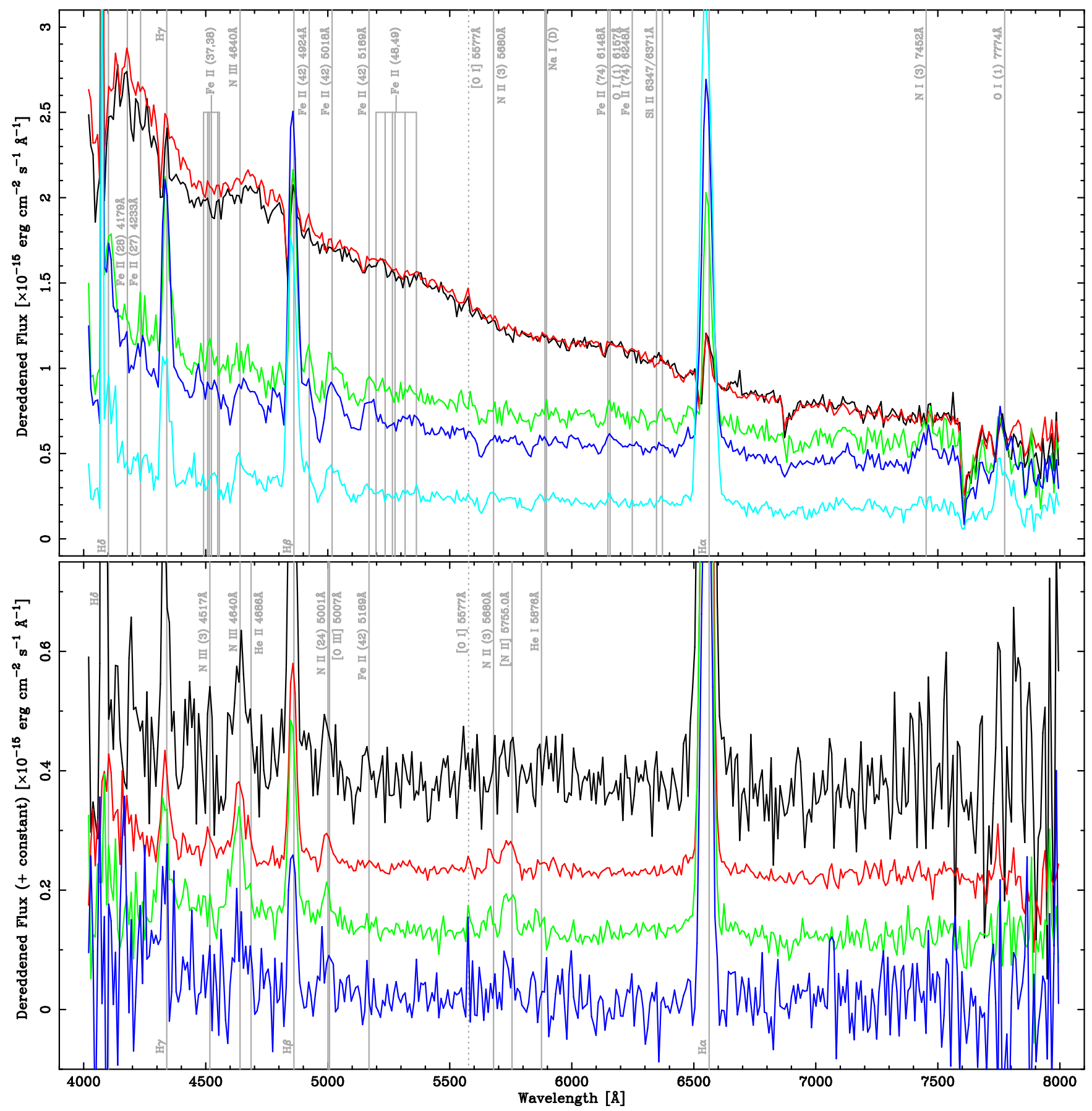

Figure 5. The Liverpool Telescope SPRAT optical spectra of nova AT 2016dah. Top: Optically thick fireball and early decline 'principle' Fe II spectra. Spectra from brightest to faintest: $2.150 \mathrm{~d}$ post-eruption (black); $3.117 \mathrm{~d}$ (red); $4.174 \mathrm{~d}$ (green); and $7.141 \mathrm{~d}$ (blue). Bottom: Transition from principle spectra to Orion phase. Brightest to faintest: $14.115 \mathrm{~d}$ post-eruption (black); $22.133 \mathrm{~d}$ (red); $24.149 \mathrm{~d}$ (green); and $28.134 \mathrm{~d}$ (blue). These four spectra have been offset in flux for clarity by integer multipliers of $0.1 \times 10^{-15} \mathrm{erg} \mathrm{cm}^{-2} \mathrm{~s}^{-1} \AA^{-1}$.

is joined by weak Si II 6347/6371 $\AA$ and Na I (D) lines. Emission from N I (3) at $\approx 7452 \AA$ also becomes visible at this time. These spectra are consistent with the nova being a member of the Fe II taxonomic spectral class (Williams 1992, 2012). The latter spectra of this sub-set show evidence for the appearance of emission from N III $4640 \AA$ (from day 4.17) and N II (3) $5680 \AA$ (from day 7.14). The apparent presence of [O I] $5577 \AA$ is most likely the residual from skysubtraction.
The next set of four spectra were collected between two-weeks and four-weeks post-eruption (see the bottom panel of Fig. 5) and span the epoch of $t_{2}$ to approximately $t_{3}$. Here, the systemic fading and flattening of the continuum has continued and the Balmer emission has weakened. The Fe II (42) emission remains at day 14.12, but no Fe II emission is evident from day 22 onward. Could the 'drop' seen in the $B$ - and $V$-band light curves around day 14 (see Section 3.3.1) be solely due to the weakening of the Fe II emission, 

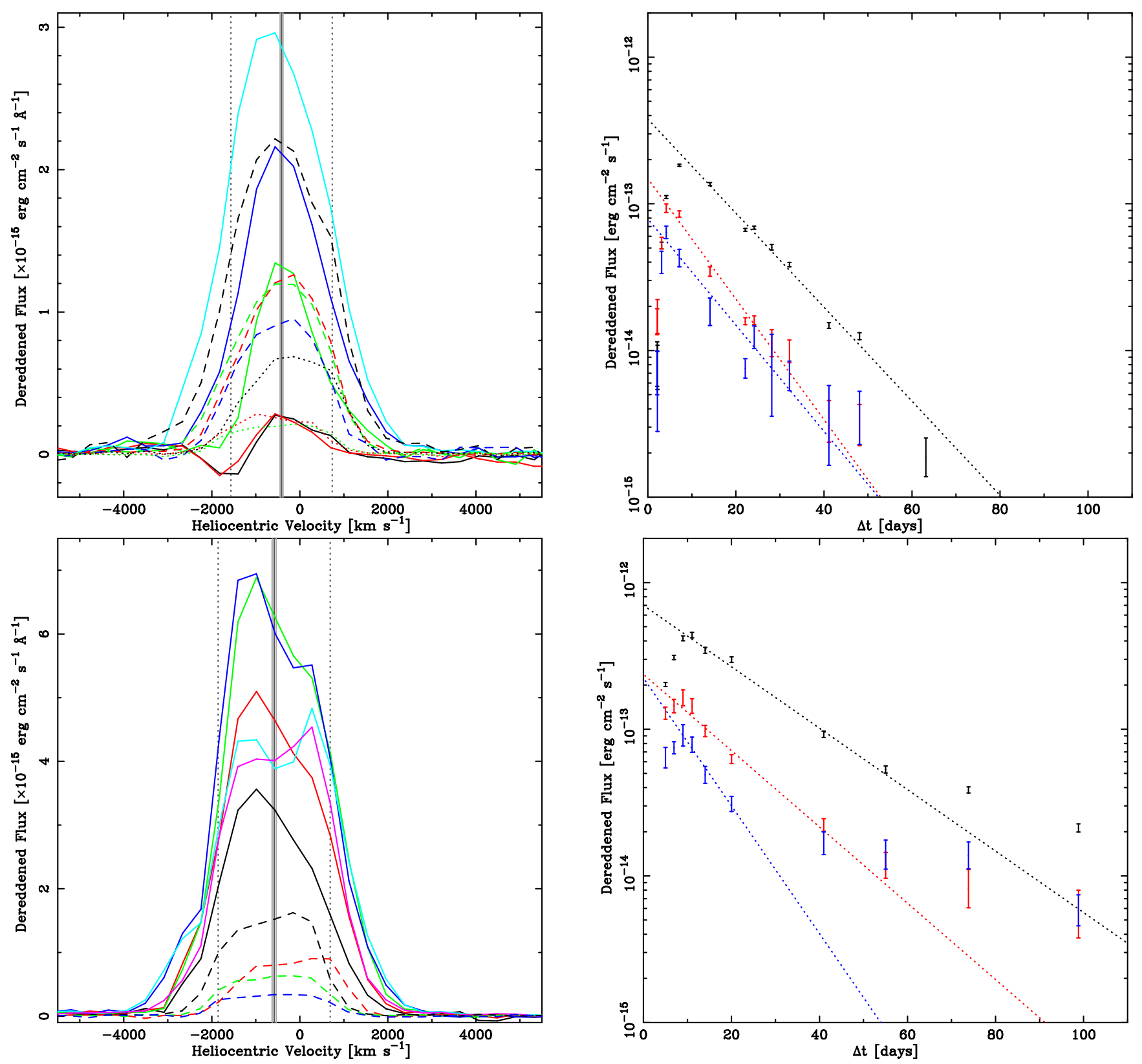

Figure 6. Left: $\mathrm{H} \alpha$ line profile evolution for AT 2016dah (top) and AT 2017fyp (bottom) following continuum subtraction and Heliocentric correction. The line colours are consistent with those used in Figs 5, 7, and 8. The solid and dashed lines relate to the spectra in the top and bottom panels of Figs 5 and 8 , respectively, the dotted lines (AT 2016dah only) relate to those spectra shown in Fig. 7. The vertical solid lines indicate the measured average line centre (and uncertainties), the vertical dotted lines indicate the extent of the measured average FWHM. Right: The flux evolution of the $\mathrm{H} \alpha$ (black), $\mathrm{H} \beta$ (red), and $\mathrm{H} \gamma$ emission lines for AT 2016dah (top) and AT 2017fyp (bottom). The dotted lines indicate linear fits to portions of those data (see text).

as the Balmer decline is smooth during this phase (see Fig. 6)? Emission lines of N II 5001, $5680 \AA$ and N III 4517, $4640 \AA$ are visible throughout this set (also see Chinetti et al. 2016b). The N II $5001 \AA$ is seen to visibly strengthen compared to the neighbouring $\mathrm{H} \beta$ line. There is evidence for the appearance of [N II] $5755 \AA$ from day 14 , along with He I $5876 \AA$. The diminishing signal-to-noise ratio in these spectra obscures any $\mathrm{O}$ I line that may still be present. There is tentative evidence for the He II $4686 \AA$ line appearing from day 14. The movement of the flux ratio of $\mathrm{H} \beta$ :N II $5001 \AA$ towards the $\mathrm{N}$ line (around half the $\mathrm{H} \beta$ flux by day 28 ) could also suggest that the [O III] $5007 \AA$ nebular line may be be present and strengthening.
The final sub-set of four spectra (see Fig. 7) were taken between day 32 and 63 post-eruption, these range from just post- $t_{3}$ to around 5 mag below peak. The continuum continues to fade, remaining just detected in the final spectrum, the Balmer flux continues to fade. Other prominent lines include the N III $4640 \AA$ feature (the Bowen-blend), and a line at around $5000 \AA$ that rivals the flux of $\mathrm{H} \beta$ and is most likely [O III] $5007 \AA$. Other lines present include He I 5876, 6678, $7065 \AA$, He II $4686 \AA$, N II $5680 \AA$ [N II] $5755 \AA$, N III (3) $4517 \AA$, and possibly [Fe II] 4244, $5159 \AA$, and [O III] $4959 \AA$. As such, AT 2016dah becomes one of a small handful of extragalactic novae to have been detected in their nebular phase. 


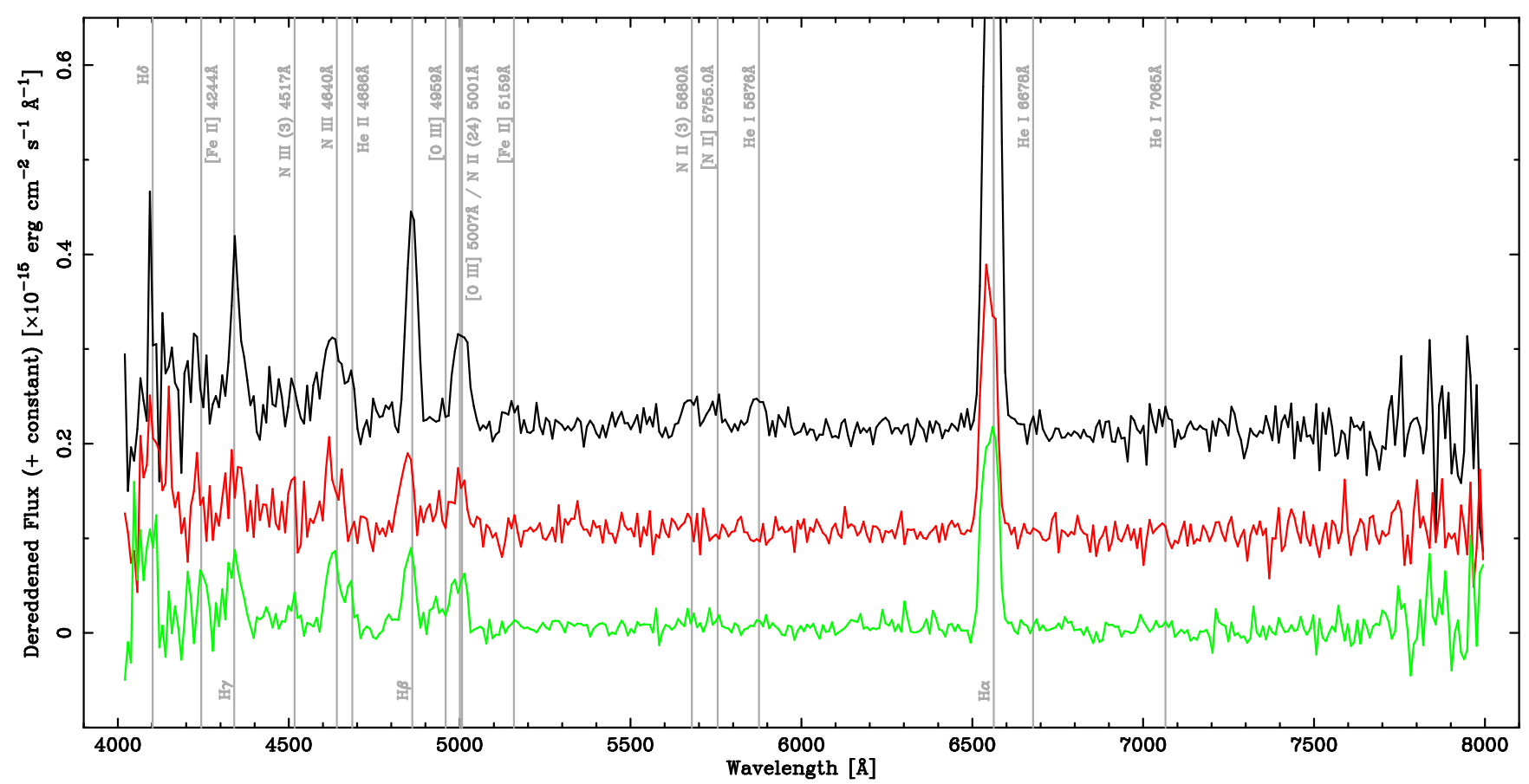

Figure 7. The Liverpool Telescope SPRAT optical spectra of nova AT 2016dah. These spectra show the nebular phase of the eruption. Brightest to faintest: $32.153 \mathrm{~d}$ post-eruption (black); $41.133 \mathrm{~d}$ (red); and $48.090 \mathrm{~d}$ (green). These three spectra have been offset in flux for clarity by integer multipliers of $0.1 \times 10^{-15} \mathrm{erg} \mathrm{cm}^{-2} \mathrm{~s}^{-1} \AA^{-1}$. The spectrum from 2016 September $13(\Delta t=63.167 \mathrm{~d})$ is not shown due to its low signal-to-noise ratio.

\subsubsection{AT 2017fyp}

The spectral coverage of AT 2017fyp began later than that of AT 2016dah. The first spectrum of this eruption was obtained $5 \mathrm{~d}$ post eruption and began a series of 11 spectra all collected by SPRAT on the LT. As discussed in Section 3.3.2, the light curve of AT 2017fyp either indicates a slow rise to peak, or alternatively an approximately flat topped profile. In either event the evolution during the first three spectra, taken on days 5, 7, and 9 (black, red, and green spectra in the top panel of Fig. 8), is consistent with the slight increase in photometric flux (particularly in the blue) seen during this stage. These three and the remaining three in this sub-set, which were taken 11,14 , and $20 \mathrm{~d}$ post-eruption (the blue, cyan, and magenta spectra, respectively), were all collected between maximum light and approximately $t_{2}$. Following the initial rise in continuum luminosity there follows a systemic decline in flux. All these spectra are dominated by Balmer emission, and there is no evidence for absorption components in the earlier spectra. This would seem to suggest that the light curve is indeed flat topped, rather than this stage being a slow rise to maximum (with the continuum still optically thick). The width of the $\mathrm{H} \alpha$ line in the first epoch is $2440 \pm 60 \mathrm{~km} \mathrm{~s}^{-1}$. Emission lines from Fe II (42) are particularly prominent; those from multiplets $26,27,37,38$, 48,49 , and 74 are also present. A line at $\sim 5530 \AA$ may be due to Fe II (55) or could be Mg I (9) $5528 \AA$. The O I (1) $7774 \AA$ line is present as is the Si II doublet. However, throughout this whole subset the following lines are also clearly present: He I 5876, $7065 \AA$, N II (3) $5680 \AA$, N III $4640 \AA$, and possibly He II $4686 \AA$ (although, at this early stage, this line may actually be O II $4650 \AA$; see for e.g. Harvey et al. 2018). As such, these spectra best fit the hybrid taxonomic class (also see Hosseinzadeh et al. 2017); although a good number of hybrids display a transition between spectral types, rather than displaying both so prominently for an extended period.
Over this period the slope of the continuum (albeit hard to define unambiguously) appears roughly consistent with that expected for optically thin free-free emission, as early as day 5 post-eruption.

Gaussian fits were again performed for the $\mathrm{H} \alpha-\mathrm{H} \gamma$ emission lines. The evolution of the Balmer line fluxes and the $\mathrm{H} \alpha$ line profile is shown in the bottom two panels of Fig. 6. As with AT 2016dah, the Balmer emission peaks after maximum light (which we assume occurred between $4 \lesssim \Delta t \lesssim 9 \mathrm{~d}$ post-eruption). Again, following a slow rise, the Balmer emission peaks at $\approx 11 \mathrm{~d}$ post-eruption. After this peak, the Balmer line emission declines with an approximately linear form until $\sim 50 \mathrm{~d}$ post-eruption where the decay rate seems to slow. The weighted average of the Heliocentric corrected line centre of $\mathrm{H} \alpha$ from all 10 spectra is $-580 \pm 50 \mathrm{~km} \mathrm{~s}^{-1}$, which corresponds to a redshift of $z=(-1.9 \pm 0.2) \times 10^{-3}$. The $\mathrm{H} \alpha$ line profile of AT 2017fyp has the 'boxy' form that is typical of He/N novae. There is no significant evolution in the line width across all 10 spectra, the (weighted) average $\mathrm{H} \alpha$ width is $2550 \pm 60 \mathrm{~km} \mathrm{~s}^{-1}$.

The final sub-set of four spectra were collected between day 41 and 99 post-eruption (the black, red, ${ }^{9}$ green, and blue spectra, respectively, in the bottom panel of Fig. 8), and range from $\sim t_{3}$ throughout the late-decline. In all four, the continuum emission appears essentially consistent, but is only marginally detected. These spectra remain dominated by the waning Balmer emission, by day 41, any Fe II emission is undetected. The most prominent lines are the Bowen-blend complex and [O III] $5007 \AA$ (again supported by the waning strength of $\mathrm{H} \beta$ relative to this line and the $4959 \AA$ line). He II $4686 \AA$ also seems to be present. He I 5876, $7065 \AA$ remain detected, as do O I (1) $7774 \AA$ and the Si II doublet (at day

\footnotetext{
${ }^{9}$ The apparent broad line, redward of He I in the $\Delta t=55 \mathrm{~d}$ spectrum, is actually the ghost image of a bright and saturated star that has persisted from a previous spectrum acquisition observation performed by SPRAT.
} 


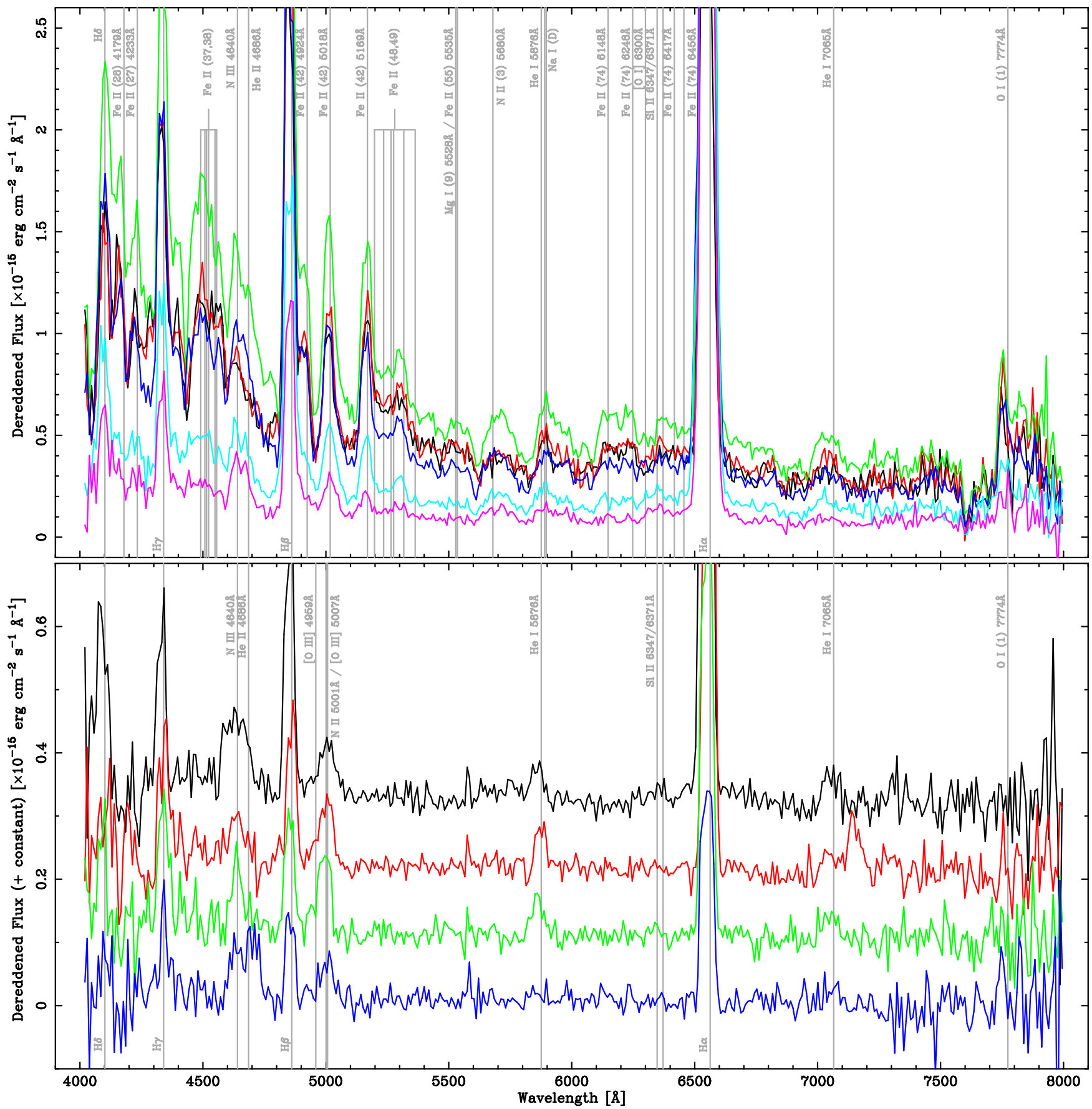

Figure 8. The Liverpool Telescope SPRAT optical spectra of nova AT 2017fyp. Top: The principle Fe II + He/N (hybrid) spectra during the early decline spectra. Spectral sequence: $5.017 \mathrm{~d}$ post-eruption (black); $6.938 \mathrm{~d}$ (red); $9.001 \mathrm{~d}$ (green); $11.052 \mathrm{~d}$ (blue); $14.037 \mathrm{~d}$ (cyan); and 20.025 d (magenta). Bottom: Transition from Orion to nebular spectra. Brightest to faintest: $40.965 \mathrm{~d}$ post-eruption (black); $55.046 \mathrm{~d}$ (red); $73.859 \mathrm{~d}$ (green); and $98.832 \mathrm{~d}$ (blue). These four spectra have been offset in flux for clarity by integer multipliers of $0.1 \times 10^{-15} \mathrm{erg} \mathrm{cm}^{-2} \mathrm{~s}^{-1} \AA^{-1}$.

41). This indicates that, as was the case for AT 2016dah, we have observed AT 2017fyp in its nebular phase.

\section{SPATIAL DISTRIBUTION}

The Andromeda Stream, or the Southern Andromeda Stream, or the M31 Giant/Great Stellar Steam was discovered by Ibata et al. (2001a, also see Ferguson et al. 2002) who employed a wide-field survey of the halo of M31 using the Isaac Newton Telescope. The
GSS appears almost linear on the sky and roughly follows a line connecting M32 and M 110 (aka NGC 205; Ibata et al. 2001a), extending to $\sim 5.5$ to the south of M 31 and $\sim 3.5$ to the east (see their fig. 23, which also indicates the main structures around M 31 Ibata et al. 2007). The GSS ranges from $\sim 100 \mathrm{kpc}$ behind M 31 at its southern-most extreme to around $30 \mathrm{kpc}$ in front of M 31 at its northern reach (McConnachie et al. 2003). Modelling by Font et al. (2006) indicated that the mass of the stream's progenitor (a satellite of M31) $>10^{8} \mathrm{M}_{\odot}-$ a massive dwarf galaxy. Ibata et al. 


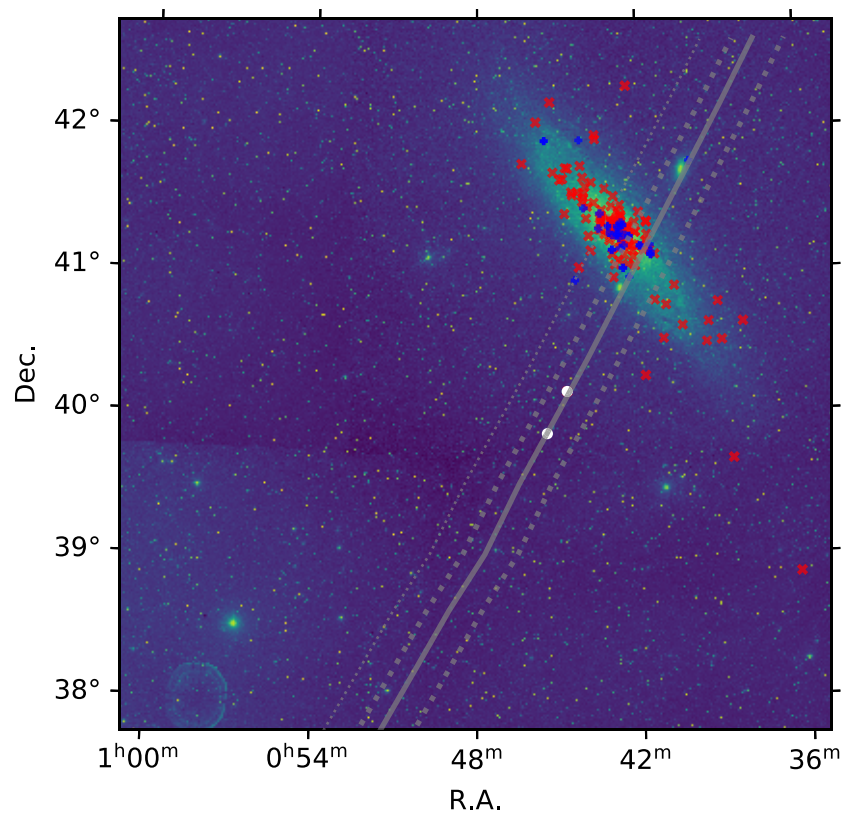

Figure 9. As Fig. 1, but showing a much wider field, with M 31 offset to the north-west. The solid grey diagonal line indicated the approximate location of the peak stellar density of the M 31 Giant Stellar Stream, the dashed grey lines delimits the bulk of the stream's stellar content, the dotted grey line indicates the lower density eastern confines of the stream. By inspection, AT 2016dah and AT 2017fyp clearly lie along the stream's central peak density region.

(2004) and Font et al. (2006) used velocity arguments to exclude an M 32 or M 110 passage of M31 as the source of the stream. The latter proposed Andromeda VIII (Morrison et al. 2003) as the potential progenitor, whereas the former suggested that And VIII may simply be part of the stream, a suggestion backed up by Merrett et al. (2006). More recently, Fardal et al. (2013) proposed that the progenitor was a 'previously unknown' M 31 satellite with stellar mass $(3.7 \pm 0.7) \times 10^{9} \mathrm{M}_{\odot}$, of order the mass of the Magellanic Clouds, and that the encounter with M31 occurred $760 \pm 50 \mathrm{Myr}$ ago.

In Fig. 9, we partially recreate those data shown initially in Fig. 1. However, the field of view has been extended from $3^{\circ} \times 3^{\circ}$ to $5^{\circ} \times$ $5^{\circ}$, with M 31 located in the north-west (top-right). Again, all M 31 novae with known spectral types from Shafter et al. (2011) and Ransome et al. (in preparation) are shown, as are the locations of AT 2016dah and AT 2017fyp, to the far south. We have overplotted the locus of the M31 GSS based upon the descriptions within the literature, particularly Ibata et al. (2001a) and McConnachie et al. (2003). Here, the solid grey line indicates the approximate line of peak stellar density along the stream, the dashed lines indicate the western edge and the region of equivalent stellar density to the east, the dotted line indicates the eastern extreme (also see Fig. 11). From inspection alone, the alignment of AT 2016dah and AT 2017fyp with the central line of the M31 GSS is remarkable.

The location of both AT 2016dah and AT 2017fyp along the line of the GSS (see Fig. 9) strongly implies that the novae are physically located within the GSS, but with the spatial extent of surveys of M 31 and the substantial number of novae detected, it is important to consider the possibility that this alignment is a chance coincidence, and the novae are unconnected with the GSS.

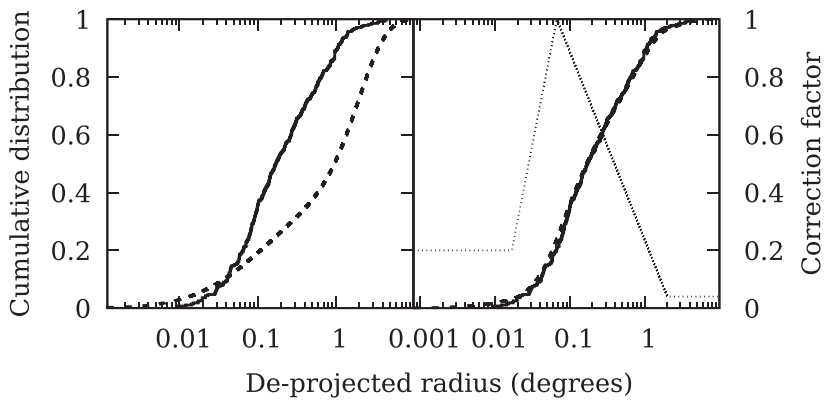

Figure 10. Comparison of the spatial distribution model to the observed nova sample. Left: the cumulative distribution in $r$ of the observed novae (solid line) is compared to the equivalent distribution from a combination of $1000 \mathrm{MC}$ iterations with a model value of $\theta=0.18$ (dashed line), without any completion correction. Right: the same observed distribution is compared to the result from MC simulations where the four-step correction function shown (thin, dotted line) is applied. A Kolmogorov (1933)-Smirnov (1948) test applied to the two sets of distributions gives a 0 per cent chance that the uncorrected simulations are drawn from the observed distribution of novae, but a 67 per cent chance that the corrected simulations are drawn from the observed distribution.

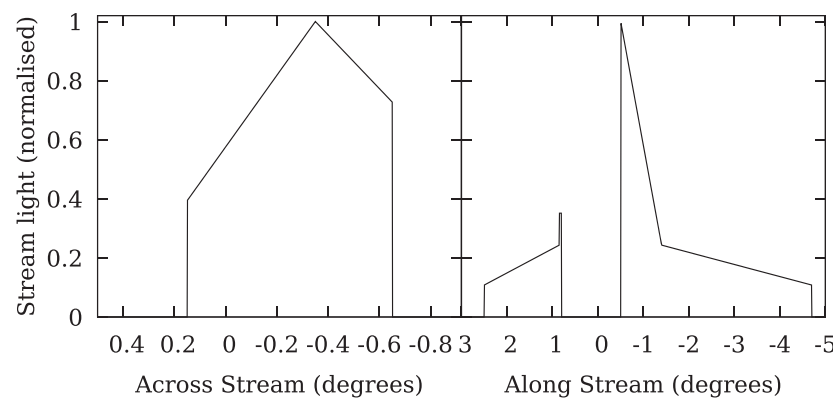

Figure 11. Our approximation to the GSS stellar light originally presented by McConnachie et al. (2003). On the left is simplified functional form we use to determine the average light along the GSS and on the right the equivalent across the GSS. The model light at any point is the product of the two functions at that position.

\subsection{Modelling the spatial distribution of a sample of novae}

We therefore require a model for the underlying distribution of nova in our sample, assuming that the GSS is not a source of novae. Armed with such a model, we can create Monte Carlo (MC) simulations of the nova distribution and explore that fraction that have a similar (or greater) association to the GSS. We adopt the approach of Darnley et al. (2006) and Williams et al. (2016, also see Ciardullo et al. 1987, Shafter \& Irby 2001, and Darnley \& Henze 2019) who define

$\Psi_{i}=\frac{\theta \mathscr{L}_{i}^{l}+\mathscr{L}_{i}^{b}}{\theta \sum_{i} \mathscr{L}_{i}^{l}+\sum_{i} \mathscr{L}_{i}^{b}}$,

where, over a grid of positions $i, \Psi$ is the probability of a nova erupting at a given location, $\theta$ is the ratio of the disc and bulge nova eruption rates per unit ( $r^{\prime}$-band) flux, and $\mathscr{L}_{i}^{b}$ and $\mathscr{L}_{i}^{d}$ the disc and bulge contributions to the ( $r^{\prime}$-band) flux at that location, respectively. Using this model Darnley et al. (2006) studied a sample of novae (see Darnley et al. 2004) close to the core of M 31 and found $\theta=$ 0.18 . Williams et al. (2016) adopted the same value of $\theta$ but, with a larger, more heterogenous sample, they found that a correction 
needed to be applied to compensate for variable completeness of the sample.

To estimate the flux component $\mathscr{L}_{i}^{b}$ we model the bulge with elliptical isophotes with an axial ratio $b / a=0.6$ (Ciardullo et al. 1987) and a standard $r^{1 / 4}$ law (de Vaucouleurs 1953). For the disc light $\mathscr{L}_{i}^{l}$, an exponential is used (Freeman 1970). Both of these models are defined along the semimajor axis of the component $\left(a_{\mathrm{b}}\right.$ and $a_{\mathrm{d}}$ for bulge and disc, respectively) yielding

$\log \left[\mathscr{L}_{\mathrm{b}}\left(a_{\mathrm{b}}\right) / \mathscr{L}_{\mathrm{b}}\right]=-3.33\left[\left(a_{\mathrm{b}} / a_{\mathrm{b}}^{0}\right)^{1 / 4}-1\right]$,

where $\mathscr{L}_{\mathrm{b}}$ is the flux at some distance $a_{\mathrm{b}}^{0}$ and is used for normalization, and

$\mathscr{L}_{\mathrm{d}}\left(a_{\mathrm{d}}\right)=\mathscr{L}_{\mathrm{d}}^{0} \mathrm{e}^{-a_{\mathrm{d}} / a_{\mathrm{d}}^{0}}$,

where $\mathscr{L}_{\mathrm{d}}^{0}$ and $a_{\mathrm{d}}^{0}$ are similarly normalization factors.

We adopt a similar approach, but, clearly, require a different correction factor for our sample, which are the spectroscopically confirmed novae in the samples from Shafter et al. (2011) and Ransome et al. (in preparation), as shown in Fig. 1. It is important to note that the purpose of this model is to produce suitable MC simulations that mirror the underlying constraints of the observed sample. There is a degeneracy between the specific value for $\theta$ used and the details of the completeness correction, so no conclusions can be drawn from the exact values of either, but as long as the observed distribution is matched, the MC simulations can be used to test the hypothesis that the GSS is not the source of any novae.

For each MC iteration, the model is used to create a probability of a nova occurring at any observed position in a fine grid, with a resolution of 4 arcsec over a large $\left(10^{\circ} \times 10^{\circ}\right)$ field, and a random number generator used to seed a single nova. The simulated nova is associated at random to either the discc or bulge (weighted appropriately by the model flux values at that point) and a deprojected radius $r$ is calculated (i.e. the equivalent semmajor axis distance). The completion factor at that radius is then used to determine whether that particular nova is 'observed' and placed into the mock catalogue for that iteration. This is repeated until the number of novae in the MC iteration matches the observed sample (276). This process is in turn repeated for a large number of MC iterations (at least 1000) to produce at least 1000 separate simulated nova 'catalogues'.

For each MC iteration, a set of $r$ values for the observed nova are generated. Since the value of $r$ for a particular nova depends upon whether it is in the bulge or disc, and that is not known for the novae, the model is used to associate a probability of being bulge or disc with, for each MC iteration, every observed nova assigned at random in line with that probability and hence an appropriate $r$ calculated. Since the MC simulations only require us to produce a distribution with the correct radial characteristics, we do not need to estimate or model the full on-sky completeness function, but just correct for the incompleteness in $r$. Therefore, the completion correction is designed to minimize the difference between the cumulative distribution in $r$ of real and simulated nova. It was generated by taking the ratio of the cumulative distributions of uncorrected and observed nova in a series of bins in $\log (r)$ and fitting a set of steps to the ratio.

This can be seen in Fig. 10 where the cumulative distribution in $r$ of the observed nova sample and combined MC iterations are compared. The correction factor comes in four steps: close to the core, completion is low; completion then increases out into the disc and is normalized to 1 at its maximum before dropping down to a low level. This matches what might be expected.

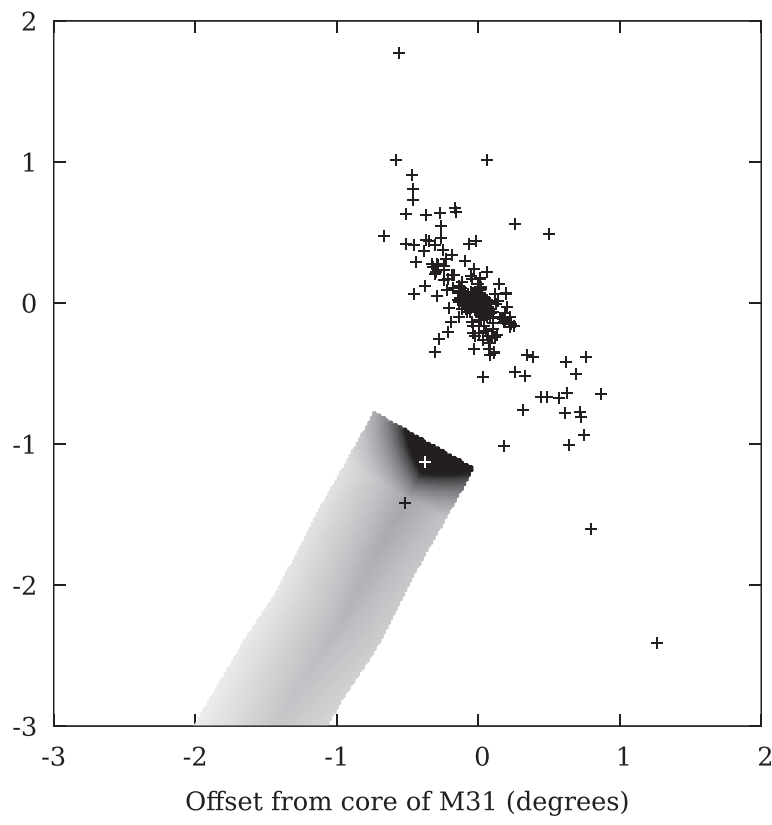

Figure 12. The simplified model of the stream light used, with the sample of observed novae shown for comparison, cf. Fig. 9.

In the inner regions, the high background makes detection and spectroscopic follow-up of novae difficult, so as the flux from the galaxy drops, the completeness increases. However, further out coverage by surveys is less complete (particularly until recently, also see Section 5.6) and so completion drops again. However, it is important to remember that, although the form of the completion correction seems sensible, it is only used ensure that the model reproduces the observed distribution: a different value of $\theta$ would result in a different correction function, but the overall results would be largely unchanged.

\subsection{Modelling the Stream}

McConnachie et al. (2003) provide a detailed description of the stellar light from the GSS, which can be used to produce a simplified model that we can apply to our observed nova sample and MC simulations. Our model follows the centre line of the GSS as determined by McConnachie et al. (2003, also see Fig. 9), but approximates the light with a two-dimensional representation along the GSS.

At each position along the GSS (i.e. roughly perpendicular to the plane of M 31), and across the GSS, the average stellar light is given by the product of the simple form shown in Fig. 11. It can be seen, however, that the component along the southern (negative distance) GSS rises sharply as it nears the plane of M 31. McConnachie et al. (2003) state that this excess is due to the GSS, and not contamination from the disc light, since it is not mirrored to the north. However, given that we are only concerned with the region where disc novae should be rare, we only consider the GSS more than $1^{\circ}$ from the plane of M 31. We also only consider the southern part of the GSS, giving the final GSS light model shown in Fig. 12.

Given this model, we can determine the association of GSS light with novae (observed or MC simulated) by summing the GSS light at the position of all novae in a given sample. Novae that fall outside the GSS will produce no contribution, while those that fall inside the GSS will contribute in proportion to the stellar light at that point. 


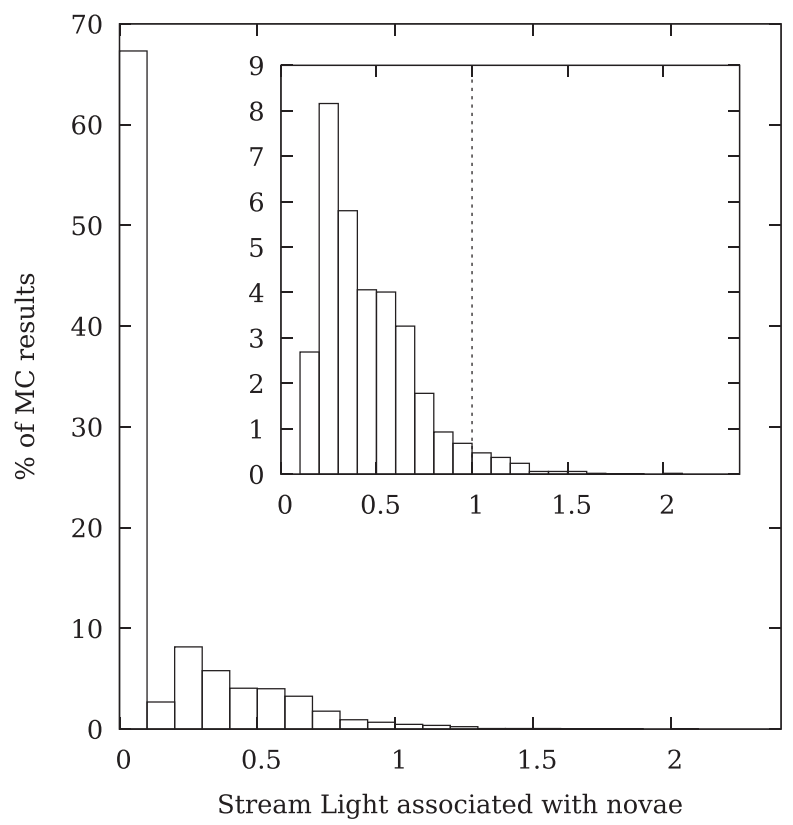

Figure 13. The histogram of GSS light associated with novae in the MC simulations. GSS light is normalised to 1 for the observed nova sample (i.e. AT 2016dah and AT 2017fyp). The inset shows the same histogram with the bin at zero GSS light removed. As can be seen, the majority of simulations have no novae on the GSS but there is a tail that stretches past 1.

For the observed sample of novae, only AT 2016dah and AT 2017fyp contribute and so we use that value to normalize the result from the MC simulated samples. The result from $10000 \mathrm{MC}$ simulations of novae distributions is given in the histogram in Fig. 13. Since the MC simulations only include the disc and bulge, any association with the GSS is random and so this gives an estimate of the likelihood that AT 2016dah and AT 2017fyp are coincidentally associated with the GSS.

Although the majority of simulations have no GSS light associated with novae, 1.3 per cent (132 out of 10000 simulations) have normalised light greater than 1 - in other words they have more GSS light associated with simulated novae than the light associated with the observed nova sample. As such, we can exclude the hypothesis that the GSS is not the source of AT 2016dah and AT 2017fyp at well beyond the $2 \sigma$ confidence limit.

\section{DISCUSSION}

As an aid to the reader, in Table 4 we provide a summary of key parameters for both AT 2016dah and 2017fyp.

\subsection{AT 2016dah}

With a $V$ band $t_{2}=7 \pm 1 \mathrm{~d}$ and exhibiting expansion velocities (inferred from the Balmer emission line FWHM and P Cygni terminal velocities) of $2300 \mathrm{~km} \mathrm{~s}^{-1}$, this rapid evolution of AT 2016dah is at the extremes of those observed for Fe II nova in M31. In their photometric and spectroscopic survey of 48 new M31 novae (91 in total), the fastest light curve evolution reported by Shafter et al. (2011) for an Fe II nova was $t_{2}(V)=8.9 \pm 0.2 \mathrm{~d}$ for the luminous nova M31N 2009-10b. However, the $\mathrm{H} \alpha$ line width of M31N 2009$10 \mathrm{~b}$ only reached $\sim 1700 \mathrm{~km} \mathrm{~s}^{-1}$ (Barsukova et al. 2009; Di Mille et al. 2009). Indeed, none of the Fe II novae reported by Shafter et al.
(2011, see their fig. 16) displayed $\mathrm{H} \alpha$ FWHMa $>2000 \mathrm{~km} \mathrm{~s}^{-1}$. Hybrid novae, those that simultaneously show elements of the $\mathrm{He} / \mathrm{N}$ and Fe II spectral classes, or evolve from one to another, are also referred to as Fe IIb or 'broad' novae (see for e.g. Della Valle \& Livio 1998). Similarly to AT 2016dah, M31N 2005-09b displayed Fe II, Na D, and He I emission, although the FWHM was only $\approx 2000 \mathrm{~km} \mathrm{~s}^{-1}$, Shafter et al. (2011) classified this system as a Fe IIb (when including the hybrid M31N 2006-10b, just 2 of 91 spectroscopically confirmed novae in M 31 at that time were hybrids or Fe IIb novae). If we turn to M33 for comparison, similarly to their M 31 work, Shafter et al. (2012) reported photometric and spectroscopic observations of eight novae in M33. Three of those novae, M33N 2003-09a, 2010-10a, and 2010-11a were classified as Fe IIb, with $\mathrm{H} \alpha$ FWHM velocities of 2700, 4210, and $2610 \mathrm{~km} \mathrm{~s}^{-1}$, respectively. Here, M33N 2003-09a and 2010-11a, which show similar velocities to AT 2016dah, displayed 'typical' Fe II spectra, where 2010-10a (like AT 2017fyp) present elements of both $\mathrm{Fe}$ II and $\mathrm{He} / \mathrm{N}$ spectra - a hybrid. It should be noted that the spectral properties of the vast majority of the Shafter et al. (2011, 2012) novae were derived from a single snapshot spectrum taken at essentially random times during their early evolution. Without detailed spectral sequences, as were obtained for both AT 2016dah and AT 2017fyp, it is always possible that a single spectrum does not reveal the whole picture of the evolution of a given nova (particularly if the contextual information provided by a well-sampled light curve is unavailable).

The Heliocentric recession velocity of AT 2016dah is $-420 \pm 30 \mathrm{~km} \mathrm{~s}^{-1}$, this is formally inconsistent (at $4 \sigma$ ) with the accepted Heliocentric recession velocity of M $31,-300 \pm 4 \mathrm{~km} \mathrm{~s}^{-1}$ (de Vaucouleurs et al. 1991). We will discuss the interpretation of this apparent discrepancy in Section 5.6.

Mooley et al. (2016) reported $15 \mathrm{GHz}$ radio observations of AT 2016dah using the Arcminute Microkelvin Imager (AMI) Large Array $\sim 25 \mathrm{~d}$ post-eruption. Those authors reported a $3 \sigma$ upper limit of $102 \mu \mathrm{Jy}$ or a spectral luminosity $<7.2 \times 10^{22} \mathrm{erg} \mathrm{s}^{-1} \mathrm{~Hz}$.

\subsection{AT 2017fyp}

AT 2017fyp displays elements of both Fe II and He/N spectra simultaneously throughout its early decline. There is no evidence, over the spectral time-series collected of a transition from one spectral type to the other, although it is possible that Fe II would have been more dominant should earlier spectra have been available. Given the spectral development and a mean FWHM of $2550 \pm 60 \mathrm{~km} \mathrm{~s}^{-1}$, AT 2017fyp is typical of a hybrid spectral type. As with the similar Fe IIb systems, hybrids appear rare in the M31 population ( $\sim 2$ per cent; Shafter et al. 2011), but appear possibly more common in younger stellar populations such as M 33 (Shafter et al. 2012).

The light curve of AT 2017fyp reveals a short lived, 5d, plateau. As such, we chose to classify AT 2017fyp as an 'F'-type or flattopped nova (after Strope et al. 2010). The definition of such novae from Strope et al. (2010) describes a flat-top that lasts 2-8 months, much longer than seen for AT 2017fyp, those authors also indicate that only a handful of Galactic novae are F-type. However, Strope et al. (2010) suggest that V2295 Ophiuchi, which exhibited a flat-top lasting $\sim 8 \mathrm{~d}$, may also fall into this class. The physical mechanism driving the flat-top phenomenon is still unclear.

AT 2017fyp shows an even greater discrepancy with the Heliocentric recession velocity of M 31 than AT 2016dah. The recession velocity of $-580 \pm 50 \mathrm{~km} \mathrm{~s}^{-1}$ differs from that of M 31 beyond $5 \sigma$. Again, this shall be discussed in Section 5.6. 
Table 4. Summary of key parameters for AT 2016dah and AT 2017fyp.

\begin{tabular}{lcc}
\hline Parameter & AT 2016dah & AT 2017fyp \\
\hline RA (J2000) & $0^{\mathrm{h}} 44^{\mathrm{m}} 41^{\mathrm{s}} .05$ & $0^{\mathrm{h}} 45^{\mathrm{m}} 25^{\mathrm{s}} .490$ \\
Dec. (J2000) & $+40^{\circ} 8^{\prime} 35^{\prime \prime} \cdot 9$ & $+39^{\circ} 50^{\prime} 52^{\prime \prime} \cdot 34$ \\
Discovery date (UT) & $2016 \mathrm{Jul} 12$ & 2017 Aug 7 \\
Time of eruption (UT) & 2016 Jul 11.96 \pm .48 & 2017 Aug $6.08 \pm 1.48$ \\
Strope et al. (2010) light-curve morphology & Smooth 'S' & 'S' or Flat topped 'F' \\
Time of maximum (days post-eruption) & 2.48 & $4-9$ \\
Peak observed apparent magnitude $\left(r^{\prime}\right)(\mathrm{mag})$ & $16.32 \pm 0.06$ & $17.041 \pm 0.007$ \\
$r^{\prime}$ band $t_{2} / t_{3}$ decline time (d) & $13.3_{-0.3}^{+0.6} / 26 \pm 2$ & $32-37 / 63-68$ \\
$V$ band $t_{2} / t_{3}$ decline time (d) & $7 \pm 1 / 13 \pm 1$ & $16-21 / 38-43$ \\
$B$ band $t_{2} / t_{3}$ decline time $(\mathrm{d})$ & $8 \pm 1 / 16_{-2}^{+3}$ & $20-25 / 53-58$ \\
Payne-Gaposchkin $(1964)$ speed class $(V$ band) & Very fast & Fast \\
Assumed reddening $E(B-V$ ) [mag] & 0.1 & 0.1 \\
Williams $(1992)$ spectral taxonomic class & Fe Irb & Hybrid \\
Radial velocity (heliocentric corrected) $\left(\mathrm{km} \mathrm{s}^{-1}\right)$ & $-420 \pm 30$ & $-580 \pm 50$ \\
H $\alpha$ FWHM $\left(\mathrm{km} \mathrm{s}^{-1}\right)$ & $2300 \pm 70$ & $2550 \pm 60$ \\
\hline
\end{tabular}

\subsection{Maximum magnitude-rate of decline}

The maximum magnitude-rate of decline (MMRD; Mclaughlin 1945) relationship has been employed for some time to estimate distances to Galactic and extragalactic novae. In recent years, the validity of that relationship has been called into question by a number of authors (see for e.g. Shara et al. 2017 and Schaefer 2018). However, Selvelli \& Gilmozzi (2019) and, more recently, Della Valle \& Izzo (2020, who performed a reanalysis of existing MMRD data) refute such claims. In either case, due to the inherent scatter, the MMRD is not a reliable distance indicator to individual novae (such as the subjects of this paper) and is strongest when used to estimate a distance towards a population of novae (see Darnley \& Henze 2019 and Della Valle \& Izzo 2020 for discussions regarding recurrent novae). The MMRD does not have the sensitivity to discriminate between individual novae in the GSS and those within M31.

Here, we simply employ the MMRD to explore whether the luminosity and decline times of AT 2016dah and AT 2017fyp are typical of the M31 population. Darnley et al. (2006) derived the only $r^{\prime}$-band MMRD for M 31 novae (see their equation 3). Using the measured $r^{\prime}$ band $t_{2}$ times for AT 2016dah and AT 2017fyp, their MMRD would predict peak apparent magnitudes of $r^{\prime}=16.2$ and 16.8, respectively. Both these MMRD predictions agree well with our observations. Additionally, the MMRD indicates that both novae are indeed of a typical luminosity for their speed class, when compared with the global M 31 population.

\subsection{X-ray emission (or lack thereof)}

AT 2016dah was observed with the XRT onboard Swift at 11 epochs between $7 \leq \Delta t \leq 87$ d post-eruption. AT 2017fyp was observed with the XRT at 10 epochs between $19 \leq \Delta t \leq 171 \mathrm{~d}$ post-eruption. No X-ray photons were detected for either source, see Table 2. At the distance of (and column towards) M31, the super-soft X-ray source (SSS) of nova eruptions are regularly detected (see, for e.g. Henze et al. 2010, 2011, 2014), although a good proportion has gone undetected despite available X-ray observations. Henze et al. (2014) observed correlations between optical and X-ray parameters for M 31 novae. These included the unveiling of the SSS $\left(t_{\mathrm{on}}\right)$ with $t_{2}$ and the expansion velocity $v_{\text {exp }}$, as estimated from optical spectra, and between the end of the SSS phase $\left(t_{\mathrm{off}}\right)$ with $t_{\mathrm{on}}$.
Utilizing equation (6) from Henze et al. (2014), we estimate the epoch of $t_{\text {on }}$ using our $r^{\prime}$-band estimates of $t_{2}$ to be: $t_{\text {on }}=70 \pm 20 \mathrm{~d}$ and $150 \pm 70 \mathrm{~d}$ post-eruption for AT 2016dah, and AT 2017fyp, respectively. Based on the $\mathrm{H} \alpha$ FWHM measurements, which is assumed to be representative of the expansion velocity, we estimate (using equation 7 from Henze et al. 2014): $t_{\text {on }} \lesssim 90$ and $t_{\text {on }} \lesssim$ $80 \mathrm{~d}$ post-eruption for AT 2016dah, and AT 2017fyp, respectively. The estimates from both methods for AT 2016dah are consistent, but given the larger expansion velocity yet slower decline of AT 2017fyp those estimates differ notably. We similarly estimate $t_{\text {off }}$ using equation (4) from Henze et al. (2014) to be in the range: $100 \lesssim$ $t_{\text {off }} \lesssim 350 \mathrm{~d}$ for AT $2016 \mathrm{dah}$, and $150 \lesssim t_{\text {off }} \lesssim 740 \mathrm{~d}$ or $t_{\text {off }} \lesssim 260 \mathrm{~d}$ for AT 2017fyp for the $t_{2}$ and $v_{\text {exp }}$ methods, respectively.

Additionally, the appearance of He II $4686 \AA$ in a nova spectrum can be an indication that part of the ejecta, or any surviving, or reformed, accretion structure is being directly illuminated by the SSS. As such, the appearance of He II often accompanies the unveiling of the SSS. For AT 2016dah, He II may have appeared as early as day 14 (but this could also be O II emission), by day 41 the identification of He II is clearer. For AT 2017fyp, He II emission appears at day 55 post-eruption. Both these epochs are consistent with the estimates using the Henze et al. (2014) relations.

Therefore it seems clear that there is a good likelihood that the Swift observations did sample the SSS phase of both AT 2016dah and AT 2017fyp. However, the SSS luminosity of these systems was below the detection limits of those observations, implying SSS luminosities $L_{\mathrm{SSS}} \lesssim 2 \times 10^{36} \mathrm{erg} \mathrm{s}^{-1}$ (sampled between 0.3 and $10 \mathrm{keV}$ ). Other possibilities include the ejecta only becoming optically thin to X-rays after the SSSs had turned-off (i.e. formally $\left.t_{\mathrm{on}}>t_{\mathrm{off}}\right)$. Or that the SSSs turned-on after both Swift campaigns had ended, given the behaviour of the Henze et al. (2014) M31 sample, this seems unlikely for such fast novae. Finally, $t_{\text {off }}$ may have occurred before the first Swift observations, i.e. before day 7 and 18 post-eruption for AT 2016dah and AT 2017fyp, respectively. However, only two novae have exhibited SSS phases so short, the recurrent novae V745 Scorpii ( $t_{\text {off }} \sim 6 \mathrm{~d}$; Page et al. 2015) and M31N 2008-12a ( $t_{\text {off }} \sim 15 \mathrm{~d}$, but only for the peculiarly late and short 2016 eruption; Henze et al. 2018). Such short SSS phases are due to a TNR on the surface of a near-Chandrasekhar mass $\mathrm{WD}$, the critical/ignition mass is low and the surface conditions particularly extreme. However, neither AT 2016dah or AT 2017fyp show any properties that would imply that they may be recurrent 
novae [for e.g. extremely fast light curve evolution, underluminous eruptions, high ejection velocities, (post-maximum) light-curve plateaus, ejecta deceleration, evolved donor, etc.; see the discussions within Pagnotta \& Schaefer 2014; Darnley 2019].

\subsection{Quiescent systems}

Darnley et al. (2012) demonstrated that the evolutionary state of the donor in a nova system could be determined by the position of that quiescent nova on a colour-magnitude diagram. This technique is particularly sensitive for those systems with giant donors, where the donor dominates the emission from the optical through to the mid/far-IR (also see Evans et al. 2014) at quiescence. Darnley et al. (2014) demonstrated that at the distance of M31 and M33 all quiescent novae with red giant donors could be recovered, with sufficiently deep and high spatial resolution imaging. Williams et al. (2016) and particularly Darnley et al. (2017b) went on to show that the accretion disc of systems with high mass transfer rates were recoverable in the Local Group by utilizing the near-UV.

In Fig. 14, we show the regions around AT 2016dah and AT 2017fyp in the McConnachie et al. (2003) CFHT data. As is shown in these images, there are no detected sources within at least 1 arcsec of either nova. Photometry at the position of the nova in the CFHT data gives the following quiescent upper limits: AT 2016dah, $V>24.8$ mag and $I>24.3 \mathrm{mag}$; AT 2017fyp, $V>24.5 \mathrm{mag}$ and $I>$ $22.4 \mathrm{mag}$. Although they provide the best UV coverage of location of the two novae, the GALEX data are not of sufficient depth or spatial resolution to be useful. At the distance of M 31, the Galactic red giant donor RNe V3890 Sagittarii and T Coronae Borealis would have $I$-band magnitudes of $\sim 21.5$ and $\sim 22.2$, respectively (Darnley et al. 2012). The depth of the CFHT data is, however, sufficient to rule out such luminous red giant donors for both these systems. Therefore it is most likely that the mass donor in both systems is a main-sequence star, although we also cannot rule out sub-giant donors for either system.

\subsection{Giant Stellar Stream novae}

Taken at face value, Fig. 9, that shows the location of AT 2016dah and AT 2017fyp with respect to the GSS, is extremely suggestive that both novae should be strongly associated with the GSS. But upon further inspection, one does notice that there are a small number of other spectroscopically confirmed novae that appear beyond the bulk of the typical M 31 bulge-disc novae. Our analysis of the M31 nova spatial distribution (see Section 4) does not definitively confirm that both AT 2016dah and AT 2017fyp are associated with the GSS. But it does indicate that the likelihood of, at least, two M 31 disc or bulge novae being spatially associated with the GSS by chance, at least as strongly as these two, is small, $\sim 1$ per cent.

Both AT 2016dah and AT 2017fyp reside within Field 7 of the CFHT targeted survey of the GSS undertaken by McConnachie et al. (2003). A radial velocity survey of the stream was reported by Ibata et al. (2004). That survey targeted 4 of the 13 McConnachie et al. (2003) fields, unfortunately it did not cover field 7, but did survey fields 6 and 8, located either side of field 7. Ibata et al. (2004) reported a strong velocity gradient along the stream. The southern most tip has similar heliocentric radial velocity to M 31 $\left(\sim-300 \mathrm{~km} \mathrm{~s}^{-1}\right)$, whereas where the stream appears to coincide with M31 it is approaching us relative to M $31\left(\sim-600 \mathrm{~km} \mathrm{~s}^{-1}\right)$. The radial velocity within field 6 was $\sim-480 \mathrm{~km} \mathrm{~s}^{-1}$. As such, given the trend of radial velocity along the stream, we would expect the radial velocity of stream stars within field 7 to lie between -480 and $-600 \mathrm{~km} \mathrm{~s}^{-1}$. The radial velocity of AT $2016 \mathrm{dah}$ and AT $2017 \mathrm{fyp}$ are $-420 \pm 30$ and $-580 \pm 50 \mathrm{~km} \mathrm{~s}^{-1}$, respectively. Our radial velocity measurements will probably suffer from additional systematic uncertainties from, for e.g. the nova ejecta geometry, ejecta self-absorption, the low spectral resolution, and possibly even the binary orbital motion. Even so, these radial velocity measurements are strongly suggestive that both novae are members of the GSS.

Neither the spatial distribution analysis nor the radial velocity result alone provide the proverbial smoking gun. But, taken together they present compelling evidence for both AT 2016dah and AT 2017fyp to be considered members of the GSS and therefore not M31 novae. As such, we will proceed under the assumption that both nova are associated with the GSS. But, with just two examples, it is hard to draw any concrete conclusions, nevertheless, one can speculate.

It is likely that the bulk of the stars associated with the GSS arose from the hitherto unidentified progenitor galaxy (see e.g. Fardal et al. 2013; Kirihara et al. 2017; Hammer et al. 2018, and references therein). Therefore, it would seem probable that both AT 2016dah and AT 2017fyp were originally also part of the GSS progenitor. Recent simulations of the GSS formation have predicted that the progenitor was a small spiral galaxy (see e.g. Fardal et al. 2013; Kirihara et al. 2017), perhaps not too unlike the present-day M33, although with a mass more akin to the Large Magellanic Cloud. As previously mentioned, Shafter et al. (2012) found significant differences between the spectroscopic properties of M 31 and M 33 novae. Fe IIb and hybrid novae, such as AT 2016dah and AT 2017fyp, respectively, are rare among the M 31 population ( $\sim 2$ per cent; Shafter et al. 2011); whereas such novae make up around half of those in M 33 (albeit it based on a sample of eight). However, the stellar mass of the GSS is substantially lower than M 33 or the LMC $\left(\sim 2.4 \times 10^{8} \mathrm{M}_{\odot}\right.$; Ibata et al. 2001a; Fardal et al. 2006), similar to that of the Local Group dwarf irregular NGC 6822 (Weldrake, de Blok \& Walter 2003). Based on the 'luminosity specific nova rate' (see Shafter et al. 2014; Darnley \& Henze 2019), the nova rate from such a stellar mass would be expected to be small: $\sim 0.1 \mathrm{yr}^{-1}$.

We should therefore ask why have two GSS novae been discovered only a year apart? In part the answer to this lies in the history of nova (and all variable and transient) surveys in and around M 31. As discussed in Section 4, the completeness of the M 31 nova catalogue, particularly the spectroscopically confirmed catalogue, has both spatial and temporal dependence. M 31 nova survey strategy has (almost) always focused on obtaining the largest amount of novae possible. In the early days, this meant focussing small fields on the bulge. As detector technology evolved, surveys expanded to cover more and more of the disc - but always with diminishing returns when considering the total nova yield (see Darnley \& Henze 2019 , for a fuller discussion). In the past decade or so, high-cadence large-fields surveys - such as those employed here: ASAS-SN and PTF/iPTF/ZTF - have been able to probe the large volume around M31, indeed the majority of the sky. So it is probably fair to say that we have only been capable of discovering (and confirming) GSS novae for around a decade. Given a nova rate of $\sim 0.1 \mathrm{yr}^{-1}, \mathrm{a}$ yield of two novae in that time would be well within expectations. As the temporal baseline of the GSS (indeed all such streams in the Local Group) grows over the coming years and decades, we would expect to detect more such novae, and hopefully address some of the questions raised here. 

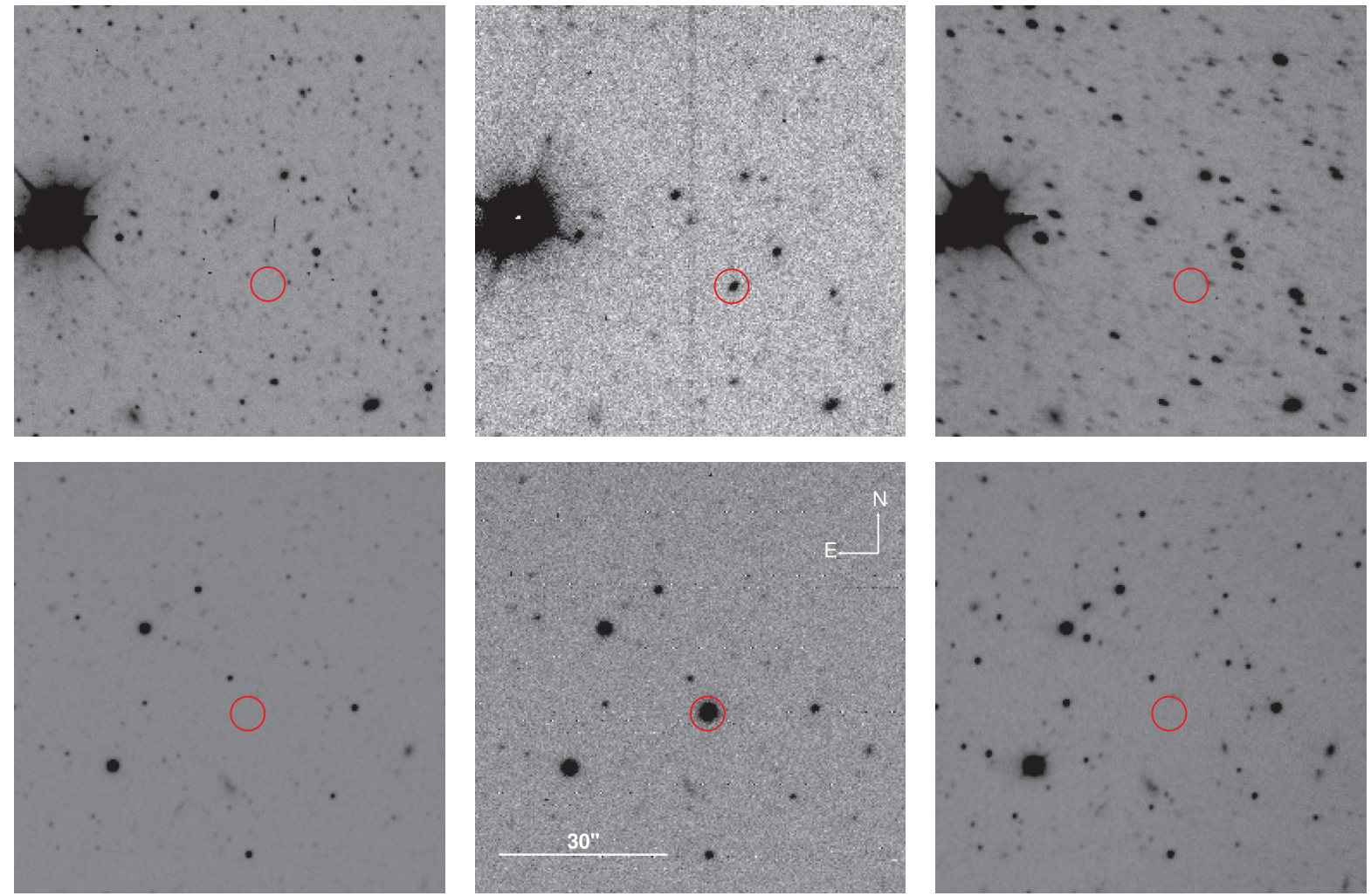

Figure 14. Progenitor search regions for AT 2016dah (top row) and AT 2017fyp (bottom row). The central image pair show Liverpool Telescope $r^{\prime}$-band eruption images $(3 \times 180 \mathrm{~s}$ exposure) with the erupting nova indicated by the red circle (radius $3 \operatorname{arcsec})$. The images to the left and right show regions of the CFHT CFH12K mosaic data $(3 \times 545 \mathrm{~s})$ around each nova (McConnachie et al. 2003), the nova position is again centred in the red circle; the left hand-image is $V$-band data, the right is $I$ band. All images are approximately a square arcminute and have the same orientation, the image scale and orientation are indicated in the bottom-centre image. The CFHT data were not of sufficient depth to recover the progenitors systems (see text for details).

\section{CONCLUSIONS}

In this section, we summarise the main findings of this paper.

(1) The classical novae AT 2016dah and AT 2017fyp are located far to the south of the bulk of the content of M31 and that host's nova population.

(2) AT 2016dah was discovered and followed photometrically and spectroscopically well before reaching maximum light. The initial spectra probe the fireball phase where the emission is dominated by a blackbody-like continuum.

(3) AT 2016dah is a very fast nova with a S-type light curve that displays prominent $\mathrm{Fe}$ II emission lines during its early decline. High ejecta velocities lead to a classification as a (rare for M31) Fe IIb nova.

(4) AT 2017fyp was a fast nova, possibly with an F-type light curve, whose early decline spectra simultaneously contained Fe II and $\mathrm{He} / \mathrm{N}$ lines, leading to a classification as a hybrid nova.

(5) Both novae were followed well into their nebular phase, in part aided by the low surface brightness so far from the centre of M31.

(6) Despite reasonable sampling by the Neil Gehrels Swift Observatory, the supersoft X-ray source of neither nova was detected. We propose that this is most likely due to the X-ray emission being below the detection limit of those observations.

(7) A progenitor search within available archival data revealed no detected quiescent counterpart for either nova. We can rule out luminous red giant donors, cf. T CrB, and, as such, we suggest that both systems are most likely to harbour main-sequence donors.

(8) Hybrid and Fe IIb novae are rare within the M31 population, perviously accounting for just 2 percent of spectroscopically confirmed novae.

Both AT 2016dah and AT 2017fyp appear strongly associated with the Giant Stellar Stream to the south of M31. The radial velocities of both novae imply an association with the GSS. The distribution of novae away from the bulge are elongated along the major axis of the inclined disc of M 31 and therefore are inconsistent with the broadly spherically symmetric halo. However, our Monte Carlo simulations of the M31 bulge and disc nova populations allows us to rule out a chance alignment of these two novae with the GSS at well beyond $2 \sigma$. Combined, this evidence leads us to claim that both novae are associated with the GSS, indeed they are the first to be associated with any tidal stellar stream. Therefore, it would seem probable that these nova systems formed within the GSS progenitor galaxy and are therefore not associated with the M31 nova population.

\section{ACKNOWLEDGEMENTS}

We would like to express our gratitude to Massimo Della Valle for his helpful and thoughtful comments when refereeing the original manuscript. The authors would like to thank Conor Ransome (and collaborators) for advanced access to the extended M31 nova spectroscopic catalogue. MJD would like to thank Kim Page for 
guidance with respect to reduction and analysis of Swift UVOT and XRT data, and Andreea Font for discussion regarding the Giant Stellar Stream. MJD and AMN also acknowledge funding from the UK Science and Technology Facilities Council (STFC) consolidated grant ST/R000484/1. ALJ acknowledges funding from INTO Newcastle University. IDWH acknowledges funding from Student Finance England. This work was supported in part by the GROWTH (Global Relay of Observatories Watching Transients Happen) project funded by the National Science Foundation Partnership in International Research and Education program under Grant No 1545949. GROWTH is a collaborative project between California Institute of Technology (USA), Pomona College (USA), San Diego State University (USA), Los Alamos National Laboratory (USA), University of Maryland College Park (USA), University of Wisconsin Milwaukee (USA), University of Washington Seattle (USA), Texas Tech University (USA), Tokyo Institute of Technology (Japan), National Central University (Taiwan), Indian Institute of Astrophysics (India), Inter-University Center for Astronomy and Astrophysics (India), Weizmann Institute of Science (Israel), The Oskar Klein Centre at Stockholm University (Sweden), Humboldt University (Germany), Liverpool John Moores University (LJMU; UK), University of Sydney (Australia). The Liverpool Telescope is operated on the island of La Palma by LJMU in the Spanish Observatorio del Roque de los Muchachos of the Instituto de Astrofísica de Canarias with financial support from STFC. IRAF is distributed by the National Optical Astronomy Observatories, which are operated by the Association of Universities for Research in Astronomy, Inc., under cooperative agreement with the National Science Foundation. This research has made use of data and software provided by the High Energy Astrophysics Science Archive Research Center (HEASARC), which is a service of the Astrophysics Science Division at NASA/GSFC. Funding for SDSS-III has been provided by the Alfred P. Sloan Foundation, the Participating Institutions, the National Science Foundation, and the U.S. Department of Energy Office of Science. The SDSS-III web site is http://www.sdss3.org. SDSS-III is managed by the Astrophysical Research Consortium for the Participating Institutions of the SDSS-III Collaboration including the University of Arizona, the Brazilian Participation Group, Brookhaven National Laboratory, Carnegie Mellon University, University of Florida, the French Participation Group, the German Participation Group, Harvard University, the Instituto de Astrofisica de Canarias, the Michigan State/Notre Dame/JINA Participation Group, Johns Hopkins University, Lawrence Berkeley National Laboratory, Max Planck Institute for Astrophysics, Max Planck Institute for Extraterrestrial Physics, New Mexico State University, New York University, Ohio State University, Pennsylvania State University, University of Portsmouth, Princeton University, the Spanish Participation Group, University of Tokyo, University of Utah, Vanderbilt University, University of Virginia, University of Washington, and Yale University. This work has made use of data from the European Space Agency (ESA) mission Gaia (https://www.cosmos.esa.int/gaia), processed by the Gaia Data Processing and Analysis Consortium (DPAC, https://www.cosm os.esa.int/web/gaia/dpac/consortium). Funding for the DPAC has been provided by national institutions, in particular the institutions participating in the Gaia Multilateral Agreement.

\section{REFERENCES}

Alam S. et al., 2015, ApJS, 219, 12

Barnsley R. M., Smith R. J., Steele I. A., 2012, Astron. Nachr., 333, 101
Barsukova E., Afanasiev V., Fabrika S., Valeev A., Hornoch K., Pietsch W., 2009, Astron. Telegram, 2251, 1

Bochanski J. J., Willman B., West A. A., Strader J., Chomiuk L., 2014, AJ, 147,76

Bode M. F., Evans A., eds, 2008, Classical Novae, 2nd Edition. Cambridge Astrophysics Series Vol. 43. Cambridge Univ. Press, Cambridge

Bode M. F., Darnley M. J., Shafter A. W., Page K. L., Smirnova O., Anupama G. C., Hilton T., 2009, ApJ, 705, 1056

Brown T. M. et al., 2013, PASP, 125, 1031

Burrows D. N. et al., 2005, Space Sci. Rev., 120, 165

Cao Y., Nugent P. E., Kasliwal M. M., 2016, PASP, 128, 114502

Chambers K. C. et al., 2016, preprint (arXiv:1612.05560)

Chinetti K., Darnley M. J., Kasliwal M. M., Mazzali P., Neill J. D., Williams S. C., 2016a, Astron. Telegram, 9248

Chinetti K., Darnley M. J., Page K. L., Williams S. C., 2016b, Astron. Telegram, 9329

Ciardullo R., Ford H. C., Neill J. D., Jacoby G. H., Shafter A. W., 1987, ApJ, 318, 520

Cuillandre J.-C., Luppino G. A., Starr B. M., Isani S., 2000, in Iye M., Moorwood A. F., eds, Proc. SPIE Conf. Ser. Vol. 4008, Optical and IR Telescope Instrumentation and Detectors. SPIE, Belligham, p. 1010

D’Onghia E., Fox A. J., 2016, ARA\&A, 54, 363

Dalcanton J. J. et al., 2015, ApJ, 814, 3

Darnley M. J., 2019, preprint (arXiv:1912.13209)

Darnley M. J., Henze M., 2019, preprint (arXiv:1909.10497)

Darnley M. J. et al., 2004, MNRAS, 353, 571

Darnley M. J. et al., 2006, MNRAS, 369, 257

Darnley M. J. et al., 2007, ApJ, 661, L45

Darnley M. J., Ribeiro V. A. R. M., Bode M. F., Hounsell R. A., Williams R. P., 2012, ApJ, 746, 61

Darnley M. J. et al., 2014, in Woudt P. A., Ribeiro V. A. R. M., eds, ASP Conf. Ser. Vol. 490, Stellar Novae: Past and Future Decades. Astron. Soc. Pac., San Francisco, p. 49

Darnley M. J. et al., 2016, ApJ, 833, 149

Darnley M. J. et al., 2017a, ApJ, 847, 35

Darnley M. J. et al., 2017b, ApJ, 849, 96

de Vaucouleurs G., 1953, MNRAS, 113, 134

de Vaucouleurs G., de Vaucouleurs A., Corwin H. G., Jr, Buta R. J., Paturel G., Fouqué P., 1991, Third Reference Catalogue of Bright Galaxies. Volume I: Explanations and references. Volume II: Data for galaxies between $0^{h}$ and $12^{h}$. Volume III: Data for galaxies between $12^{h}$ and $24^{h}$. Springer, New York

Della Valle M., Izzo L., 2020, preprint (arXiv:2004.06540)

Della Valle M., Livio M., 1998, ApJ, 506, 818

Di Mille F. et al., 2009, Astron. Telegram, 2248, 1

Dressler A., 1984, ARA\&A, 22, 185

Evans A., Gehrz R. D., Woodward C. E., Helton L. A., 2014, MNRAS, 444, 1683

Evans P. A. et al., 2009, MNRAS, 397, 1177

Fardal M. A., Babul A., Geehan J. J., Guhathakurta P., 2006, MNRAS, 366, 1012

Fardal M. A. et al., 2013, MNRAS, 434, 2779

Feldmeier J. J., Ciardullo R., Jacoby G. H., 1998, ApJ, 503, 109

Ferguson A. M. N., Irwin M. J., Ibata R. A., Lewis G. F., Tanvir N. R., 2002, AJ, 124, 1452

Font A. S., Johnston K. V., Guhathakurta P., Majewski S. R., Rich R. M., 2006, AJ, 131, 1436

Freedman W. L. et al., 2001, ApJ, 553, 47

Freeman K., 1970, ApJ, 160, 811

Gal-Yam A., Maoz D., Guhathakurta P., Filippenko A. V., 2003, AJ, 125, 1087

Gehrels N. et al., 2004, ApJ, 611, 1005

Gerhard O., Arnaboldi M., Freeman K. C., Kashikawa N., Okamura S., Yasuda N., 2005, ApJ, 621, L93

Graham M. L., Sand D. J., Zaritsky D., Pritchet C. J., 2015, ApJ, 807, 83

Güver T., Özel F., 2009, MNRAS, 400, 2050

Hachisu I., Kato M., 2006, ApJS, 167, 59 
Hammer F., Yang Y. B., Wang J. L., Ibata R., Flores H., Puech M., 2018, MNRAS, 475, 2754

Harvey E. J., Redman M. P., Darnley M. J., Williams S. C., Berdyugin A., Piirola V. E., Fitzgerald K. P., O’Connor E. G. P., 2018, A\&A, 611, A3

Healy M. W. et al., 2019, MNRAS, 486, 4334

Henze M. et al., 2010, A\&A, 523, A89

Henze M. et al., 2011, A\&A, 533, A52

Henze M. et al., 2014, A\&A, 563, A2

Henze M. et al., 2018, ApJ, 857, 68

HI4PI Collaboration, 2016, A\&A, 594, A116

Hosseinzadeh G., Valenti S., Arcavi I., Howell D. A., McCully C., 2017, Astron. Telegram, 10628, 1

Ibata R., Irwin M., Lewis G., Ferguson A. M. N., Tanvir N., 2001a, Nature, 412,49

Ibata R., Irwin M., Lewis G. F., Stolte A., 2001b, ApJ, 547, L133

Ibata R., Chapman S., Ferguson A. M. N., Irwin M., Lewis G., McConnachie A., 2004, MNRAS, 351, 117

Ibata R., Martin N. F., Irwin M., Chapman S., Ferguson A. M. N., Lewis G. F., McConnachie A. W., 2007, ApJ, 671, 1591

Jester S. et al., 2005, AJ, 130, 873

Kirihara T., Miki Y., Mori M., Kawaguchi T., Rich R. M., 2017, MNRAS, 464,3509

Kochanek C. S. et al., 2017, PASP, 129, 104502

Kolmogorov A., 1933, Inst. Ital. Attuari., 4, 1

Lasker B. M., Sturch C. R., McLean B. J., Russell J. L., Jenkner H., Shara M. M., 1990, AJ, 99, 2019

Longobardi A., Arnaboldi M., Gerhard O., Coccato L., Okamura S., Freeman K. C., 2013, A\&A, 558, A42

Martin D. C. et al., 2005, ApJ, 619, L1

Masci F. J. et al., 2017, PASP, 129, 014002

McConnachie A. W., Irwin M. J., Ibata R. A., Ferguson A. M. N., Lewis G. F., Tanvir N., 2003, MNRAS, 343, 1335

McGee S. L., Balogh M. L., 2010, MNRAS, 403, L79

Mclaughlin D. B., 1945, PASP, 57, 69

Merrett H. R. et al., 2006, MNRAS, 369, 120

Mihos J. C., Harding P., Feldmeier J. J., Rudick C., Janowiecki S., Morrison H., Slater C., Watkins A., 2017, ApJ, 834, 16

Miller G. E., 1983, ApJ, 268, 495

Mooley K. P. et al., 2016, Astron. Telegram, 9382

Morrison H. L., Harding P., Hurley-Keller D., Jacoby G., 2003, ApJ, 596, L183

Neill J. D., Shara M. M., Oegerle W. R., 2005, ApJ, 618, 692

Nicolas J. et al., 2016, Astron. Telegram, 9245

Page K. L. et al., 2015, MNRAS, 454, 3108

Pagnotta A., Schaefer B. E., 2014, ApJ, 788, 164

Payne-Gaposchkin C., 1964, The Galactic Novae. Dover Publication, New York

Piascik A. S., Steele I. A., Bates S. D., Mottram C. J., Smith R. J., Barnsley R. M., Bolton B., 2014, in Suzanne K. R., Ian S. M., Takami H., eds, Proc. SPIE Conf. Ser. Vol. 9147, Ground-based and Airborne Instrumentation for Astronomy V. SPIE, Bellingham, p. 8

Roming P. W. A. et al., 2005, Space Sci. Rev., 120, 95

Sand D. J. et al., 2011, ApJ, 729, 142

Schaefer B. E., 2018, MNRAS, 481, 3033

Schlegel D. J., Finkbeiner D. P., Davis M., 1998, ApJ, 500, 525

Selvelli P., Gilmozzi R., 2019, A\&A, 622, A186

Shafter A. W., 2019, Extragalactic Novae; A Historical Perspective. IOP ebooks. Bristol, IOP Publishing, UK

Shafter A. W., Irby B. K., 2001, ApJ, 563, 749
Shafter A. W. et al., 2011, ApJ, 734, 12

Shafter A. W., Darnley M. J., Bode M. F., Ciardullo R., 2012, ApJ, 752, 156

Shafter A. W., Curtin C., Pritchet C. J., Bode M. F., Darnley M. J., 2014, in Woudt P. A., Ribeiro V. A. R. M., eds, ASP Conf. Ser. Vol. 490, Stellar Novae: Past and Future Decades. Astron. Soc. Pac., San Francisco, p. 77 Shappee B. J. et al., 2014, ApJ, 788, 48

Shara M. M., 2006, AJ, 131, 2980

Shara M. M. et al., 2017, ApJ, 839, 109

Smirnov N., 1948, Ann. Math. Statist., 19, 279

Smith R., Steele I., 2017, Liverpool Telescope Technical Note 1: Telescope and IO:O Throughput, http://researchonline.ljmu.ac.uk/id/eprint/5699/

Stark A. A., Gammie C. F., Wilson R. W., Bally J., Linke R. A., Heiles C., Hurwitz M., 1992, ApJS, 79, 77

Steele I. A. et al., 2004, in Oschmann J. M., Jr, ed., Proc. SPIE Conf. Ser. Vol. 5489, Ground-based Telescopes. SPIE, Belligham, p. 679

Stone R. P. S., 1977, ApJ, 218, 767

Strope R. J., Schaefer B. E., Henden A. A., 2010, AJ, 140, 34

Teyssier M., Johnston K. V., Shara M. M., 2009, ApJ, 707, L22

Theuns T., Warren S. J., 1997, MNRAS, 284, L11

Tody D., 1993, in Hanisch R. J., Brissenden R. J. V., Barnes J., eds, ASP Conf. Ser. Vol. 52, Astronomical Data Analysis Software and Systems II. Astron. Soc. Pac., San Francisco, p. 173

Tonry J., Stalder B., Denneau L., Heinze A., Weiland H., Rest A., Smith K. W., Smartt S. J., 2017, Transient Name Server Discovery Report, 2017-852

Toomre A., Toomre J., 1972, ApJ, 178, 623

Weldrake D. T. F., de Blok W. J. G., Walter F., 2003, MNRAS, 340, 12

Williams R. E., 1992, AJ, 104, 725

Williams R., 2012, AJ, 144, 98

Williams S. C., Darnley M. J., Bode M. F., Keen A., Shafter A. W., 2014, ApJS, 213, 10

Williams S. C., Darnley M. J., Bode M. F., Shafter A. W., 2016, ApJ, 817, 143

Williams S. C., Darnley M. J., Henze M., 2017, MNRAS, 472, 1300

Woudt P. A., Ribeiro V. A. R. M.eds, 2014, ASP Conf. Ser. Vol. 490, Stella Novae: Past and Future Decades. Astron. Soc. Pac., San Francisco

Wright A. E., Barlow M. J., 1975, MNRAS, 170, 41

\section{SUPPORTING INFORMATION}

Supplementary data are available at MNRAS online.

Table S1. Pan-STARRS sources used for calibration of the AT 2016dah photometry.

Table S2. SDSS sources used for calibration of the AT 2017fyp photometry.

Table S3. Photometry of nova AT 2016dah as obtained by ASASSN (also see Nicolas et al. 2016), iPTF, and the LT.

Table S4. Photometry of nova AT 2017fyp as obtained by the LT, LCO, Gaia, and ATLAS (see Tonry et al. 2017).

Please note: Oxford University Press is not responsible for the content or functionality of any supporting materials supplied by the authors. Any queries (other than missing material) should be directed to the corresponding author for the article.

This paper has been typeset from a $\mathrm{T}_{\mathrm{E}} \mathrm{X} / \mathrm{LT} \mathrm{E} \mathrm{X}$ file prepared by the author. 